DIAS-STP-15-07

IMPERIAL-TP-2015-MJD-01

\title{
Gravity as the square of Yang-Mills?
}

\author{
L. Borsten ${ }^{1}$ and M. J. Duff ${ }^{2}$ \\ ${ }^{1}$ School of Theoretical Physics, Dublin Institute for Advanced Studies, \\ 10 Burlington Road, Dublin 4, Ireland \\ 2 Theoretical Physics, Blackett Laboratory, Imperial College London, \\ London SW7 2AZ, United Kingdom \\ leron@stp.dias.ie \\ m.duff@imperial.ac.uk
}

\begin{abstract}
In these lectures we review how the symmetries of gravitational theories may be regarded as originating from those of "Yang-Mills squared". We begin by motivating the idea that certain aspects of gravitational theories can be captured by the product, in some sense, of two distinct Yang-Mills theories, particularly in the context of scattering amplitudes. We then introduce a concrete dictionary for the covariant fields of (super)gravity in terms of the product of two (super) Yang-Mills theories. The dictionary implies that the symmetries of each (super) Yang-Mills factor generate the symmetries of the corresponding (super)gravity theory: general covariance, $p$-form gauge invariance, local Lorentz invariance, local supersymmetry, Rsymmetry and U-duality.
\end{abstract}

\footnotetext{
${ }^{1}$ Lectures delivered by M. J. Duff
} 


\section{Contents}

1 MJD: Tribute to Dick Arnowitt

2 Introduction 2

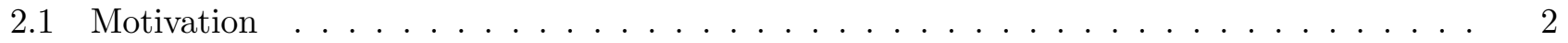

3 Covariant field dictionary 4

$4 \mathcal{N}=1$ supergravity

5 Extended supersymmetry and U-duality

5.1 Division algebras and the magic square . . . . . . . . . . . . . . . . 9

5.2 Division algebras and Yang-Mills theories . . . . . . . . . . . . . 13 


\section{MJD: Tribute to Dick Arnowitt}

It was thanks to Dick Arnowitt that I spent eleven wonderful years 1988-1999 here at Texas A\&M. My wife Lesley and I have not forgotten the kindness shown by Dick and Young-In when we first arrived here from England. It was a privilege to work in the first rate Theoretical Physics Group that Dick had built.

\section{Introduction}

The idea that gravitational physics can be understood in terms of gauge theory has reoccured a number of times, in a variety of guises. The most conceptually straight-forward approach is to regard gravity as the gauge theory of Lorentz, Poincaré or de Sitter symmetries [1 5]. The holographic principle [6, 7], concretely realised through the AdS/CFT correspondence [8 10], represents a more subtle realisation of this notion, with profound consequences for our understanding of both gauge and gravity theories. Here we appeal to a third and, at least superficially, independent incarnation:

$$
\text { gravity }=\text { Yang-Mills } \times \text { Yang-Mills. }
$$

At first sight this is a radical proposal; Einstein's general relativity describes gravity as the dynamics of spacetime, while Yang-Mills theories, as used to describe the strong, weak and electromagnetic forces, play out on spacetime. General relativity and Yang-Mills theory are seemingly worlds apart in almost every regard, from their fundamental degrees freedom to their basic symmetries. In particular, the Yang-Mills theories underlying the standard model are renormalisable, predictive quantum field theories, in stark contrast to perturbative quantum gravity.

Despite their differences, however, there already exist some fascinating hints that gravity, at least in some regimes, may be related to the square of Yang-Mills theory. String theory provided the first example in the form of the Kawai-Lewellen-Tye (KLT) relations, which connect tree-level amplitudes of closed strings to sums of products of open string amplitudes 11. More recently, invoking BernCarrasco-Johansson (BCJ) colour-kinematic duality [12] it has been conjectured [13] that the on-mass-shell momentum-space scattering amplitudes for gravity are the "double-copy" of gluon scattering amplitudes in Yang-Mills theory to all orders in perturbation theory.

The recent renaissance in amplitude calculations has been principally driven by the "on-shell paradigm". Starting with Lagrangian field theory we learnt how to compute simple amplitudes to low orders in perturbation theory. The factorial growth in complexity with loop order quickly renders traditional approaches impractical. Searching for computational efficiency, over time various generic amplitude structures (onshell recursion relations, generalised unitarity cuts, Grassmannians, scattering equations ....) were uncovered, eventually allowing the Lagrangian ladder to be kicked away. For an overview of these developments see [14. This freedom led to the discovery of new features of amplitudes, not visible from the original Lagrangian perspective. BCJ colour-kinematic duality falls into this class of surprises. Conversely, having climbed so high we can no longer see where we can from; the full significance and implications of BCJ duality remain unclear. Can we climb back down by some other route and understand the origin of these remarkable dualities? The basic idea reviewed here is to build a dictionary expressing the covariant fields of (super) gravity as the product, in a well-defined sense, of two arbitrary (super) Yang-Mills theories.

\subsection{Motivation}

We begin by sketching the BCJ colour-Kinematic duality and the double copy procedure $12,13,15$. For a more detailed account of this topic the reader is referred to the reviews [14, 16. This will not only better motivate (2.1), but also inform our field theory constructions in the subsequent sections.

Let us consider the $n$-point $L$-loop amplitude of Yang-Mills theory with an arbitrary gauge group. Converting all four-point contact terms into $s, t$ or $u$ channel trivalent pole diagrams by inserting propa- 
gators $1=s / s=t / t=u / u$, we have,

$$
A_{n}^{L}=i^{L} g^{n-2+2 L} \sum_{i \in \text { trivalent graphs }} \int \prod_{l=1}^{L} \frac{d^{D} p_{l}}{(2 \pi)^{D} S_{i}} \frac{c_{i} n_{i}}{\prod_{a_{i}} p_{a_{i}}^{2}} .
$$

The sum is over all $n$-point $L$-loop graphs $i$ with only trivalent vertices. $c_{i}$ denotes the kinematic factor of graph $i$, composed of gauge group structure constants. $n_{i}$ denotes the kinematic factor of graph $i$. It is a polynomial of Lorentz-invariant contractions of polarisation vectors and momenta. The $p_{a_{i}}^{2}$ are the propagators for each graph $i . S_{i}$ is the dimension of the automorphism group of graph $i$.

The set of $n$-point trivalent graphs can be organised into triples $i, j, k$ such that they differ in only one propagator. For such a triple the three disctint propagators are embedded in the same graph, connected to the same four incoming edges, but in the $s, t, u$ channel for (say) $i, j, k$, respectively. For such a triple the colour factors will obey a Jacobi identity

$$
c_{i}+c_{j}+c_{k}=0
$$

and consequently the generalised gauge transformations

$$
n_{i} \rightarrow n_{i}+s \Delta, \quad n_{j} \rightarrow n_{j}+t \Delta, \quad n_{k} \rightarrow n_{k}+u \Delta
$$

leave the amplitude 2.2 invariant 12 . It was proposed in 12 that one can arrange the diagrams, using the generalised gauge transformations if necessary, to display a colour-kinematic duality:

$$
c_{i}+c_{j}+c_{k}=0 \Rightarrow n_{i}+n_{j}+n_{k}=0
$$

and if $c_{i} \rightarrow-c_{i}$ under the interchange of two legs then $n_{i} \rightarrow-n_{i}$. A reorganisation admitting this surprising relationship between colour and kinematic data was shown to exist for all $n$-point tree-level amplitudes in [15]. Although there is as yet no proof, the colour-kinematic duality is conjectured to hold, with highly non-trivial evidence [17,18, at any loop level, thus going beyond the KLT relations [12,13]. While it is clear that the colour factors should obey Jacobi identities (by definition), it is not at all obvious that the kinematic factors should play by the same rules!

This suggests that there is in fact some underlying kinematic algebra mirroring the properties of conventional Lie algebras, as described in [19,20]. In general, this hidden algebra cannot be made manifest at the Lagrangian level, however for the self-dual sector it can be identified as a diffeomorphism Lie algebra, which determines the kinematic numerators of generic tree-level maximally helicity violating amplitudes [19]. Important features of the BCJ construction can also be derived from string theory. In particular, the BCJ relations 12 (which we have not discussed) have been obtained via monodromy relations 21 23. Moreover, explicit expressions for colour-kinematic duality respecting local tree-level numerators at $n$-points have been derived using the pure spinor approach to string theory amplitudes and dimensional reduction 24,25]. The string theoretic approach is also suited to loop level calculations. For example, colour-kinematic duality respecting numerators at five points with one and two loops were obtained in 26, 27, while at arbitrary multiplicity one-loop maximally helicity violating colour-kinematic duality respecting numerators have been constructed in 28].

More remarkable still is the double-copy prescription [12,13, 15]. Assuming one has found a colourkinematic duality respecting representation of the $n$-point $L$-loop gluon amplitude, the equivalent $n$-point $L$-loop graviton amplitude is obtained by simply replacing each colour factor, $c_{i}$, with a second kinematic factor, $\tilde{n}_{i}$, as depicted in Figure 1. Examining the unitary cuts of the gravity amplitude obtained via the double-copy is sufficient to prove it reproduces the correct result, assuming colour-kinematic duality is satisfied in one of the Yang-Mills factors. These ideas are seamlessly extended to supersymmetric theories. In particular, the square of the amplitudes of the maximally supersymmetric $\mathcal{N}=4$ super Yang-Mills theory yield amplitudes of the maximally supersymmetric $\mathcal{N}=8$ supergravity theory. 


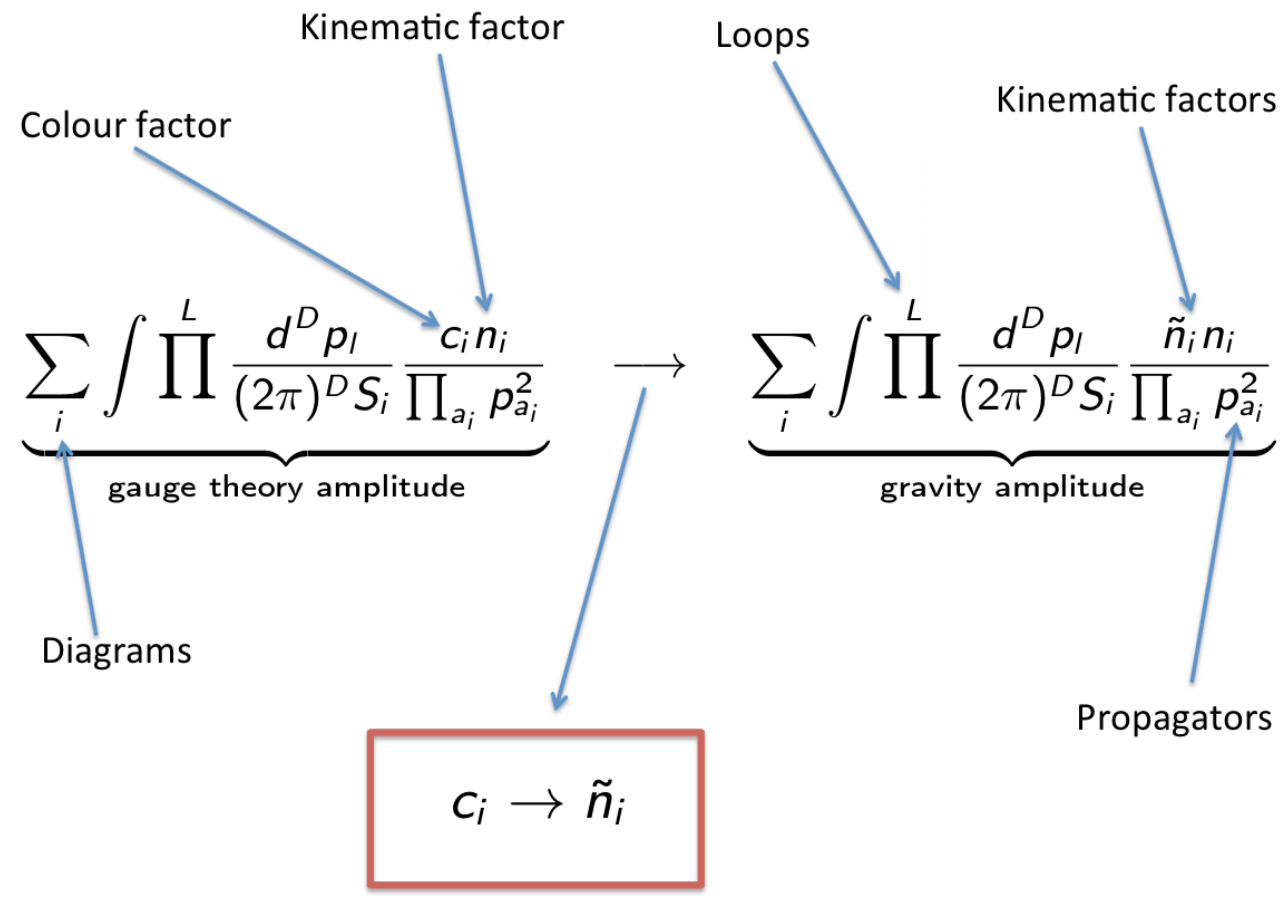

Figure 1: The double-copy procedure. Assuming the gauge theory amplitude on the left has been arranged to display colour-kinematic duality then the gravity amplitude on the right is straight-forwardly obtained by replacing the colour factors with a second copy of the kinematic factors. Note, the second factor does not have correspond to the same Yang-Mills theory. The (supressed) Yang-Mills coupling constants must be replaced by the gravitational coupling constant $g \rightarrow \kappa / 2$, where $\kappa^{2}=16 \pi G_{N}$.

The double-copy picture is not only conceptually compelling but also computationally powerful, bringing previously intractable calculations with in reach. This has pushed forward dramatically our understanding of divergences in perturbative quantum gravity, revealing a number of unexpected features and calling into question previously accepted arguments regarding finiteness. For instance, the four-point graviton amplitude in $\mathcal{N}=8$ supergravity has been shown to be finite to four loops [17], contradicting some early expectations $\left[29\right.$. In particular, the existence of a supersymmetric $R^{4}$ counter-term in $\mathcal{N}=1$ supergravity at three loops was established in [30], although it was already noted there that this result may not hold when more supersymmetry is considered. It has since been shown that the four-loop cancellation can be accounted for by supersymmetry and $E_{7(7)}$ U-duality 31 35. The consensus, however, is that at seven loops any would-be cancellations cannot be "consequences of supersymmetry in any conventional sense" 31. Unfortunately, seven loops in $\mathcal{N}=8$ supergravity remains beyond reach but by decreasing the amount of supersymmetry these arguments apply at lower loop order. Indeed, the four-point amplitude of $D=4, \mathcal{N}=5$ supergravity has been shown to be finite to four loops, contrary to all expectations based on standard symmetry arguments [18]. There are "enhanced cancellations" at work and the conclusion that $\mathcal{N}=8$ supergravity will diverge at seven loops is thrown into doubt. Although the majority opinion is that $\mathcal{N}=8$ supergravity will diverge at some loop order, there is something deeper at work we have yet to understand fully and question remains very much open.

\section{Covariant field dictionary}

These developments raise the question: to what extent, or in what sense, can one regard gravity as the square of Yang-Mills? Is there a deeper connection underlying the amplitude relations? One approach to addressing such questions is to build a dictionary at the level of fields, as opposed to on-shell states 
or amplitudes. In a sense this runs contrary to the "on-shell paradigm" that took us here. Going back off-shell may nonetheless be instructive. This approach has been examined at the level of Lagrangians in 13, 19 and classical solutions in [36 38. Here instead we focus on expressing the covariant fields of (super)gravity in terms of the product of (super) Yang-Mills fields. The first consistency check such a dictionary must pass is at the level of symmetries. As we shall review, the gravitational symmetries of general covariance, $p$-form gauge invariance, local supersymmetry and local chiral symmetry, R-symmetry and U-duality follow from those of Yang-Mills at linearised approximation.

Much of the squaring literature invokes a mysterious product:

$$
A_{\mu}(x) " \otimes " \tilde{A}_{\nu}(x)
$$

Here, $A_{\mu}$ and $\tilde{A}_{\nu}$ are the gauge potentials of two distinct Yang-Mills theories, which we will refer to as left (no tilde) and right (tilde), respectively. They can have arbitrary and independent non-Abelian gauge groups $G$ and $\tilde{G}$. Reading off the meaning of " $\otimes$ " from the tensor product branching rules of the appropriate spacetime little group representations or corresponding string states one can consistently match the symmetries. See in particular [39,40]. Here, we are instead seeking a concrete definition of " $\otimes$ " at the level of field theory which is valid whether or not there is an underlying string interpretation. This raises two immediate questions: (i) where do the gauge indices go? (ii) does it obey the Leibnitz rule?

Guided by the structure of the amplitude relations and requirements of symmetry we introduced a covariant product rule in [41]:

$$
f " \otimes " g:=f \star \Phi \star g \text {. }
$$

Let us review the ingredients in $(3.2)$. The $\star$ product denotes a convolutive inner tensor product with respect to the Poincaré group combined with a Killing form $\langle\rangle:, \mathfrak{g} \otimes \mathfrak{g} \rightarrow \mathbb{R}$,

$$
[f \star g](x)=\int d^{D} y\langle f(y), g(x-y)\rangle \text {. }
$$

We have further introduced the "spectator" field $\Phi$, a $G \times \tilde{G}$ bi-adjoint valued scalar. The convolution reflects the fact that the amplitude relations are multiplicative in momentum space. It turns out to be essential for reproducing the local symmetries of (super)gravity from those of the two (super) Yang-Mills factors. The Killing form accounts for the gauge groups, while the spectator field allows for arbitrary and independent $G$ and $\tilde{G}$. It fact, the appearance of $\Phi$ is quite natural from the perspective of amplitude relations. Its necessity was identified by Hodges in the context of twistor-theory [42]. From the perspective presented in subsection 2.1, rather than sending $c_{i} \rightarrow \tilde{n}_{i}$, doubling the kinematics and removing the colour, one could also send $n_{i} \rightarrow \tilde{c}_{i}$, doubling the colour and removing the kinematics. In [43, 44 this was shown at tree-level to yield the amplitudes of a global $G \times \tilde{G}$ bi-adjoint scalar field theory with cubic interaction term,

$$
\mathcal{L}_{\text {int }}=-f_{i j k} \tilde{f}_{i^{\prime} j^{\prime} k^{\prime}} \Phi^{i i^{\prime}} \Phi^{j j^{\prime}} \Phi^{k k^{\prime}}
$$

The transformation rules of $\Phi$ are fixed by this theory. A scalar field also appeared independently, but in close analogy to our spectator field, in the double-copy construction of Kerr-Schild gravity solutions from Yang-Mills solutions in 36.

\section{$4 \mathcal{N}=1$ supergravity}

Having introduced the covariant product, let us now work through the simplest example exhibiting all the local symmetries of interest. We consider the product of a left $\mathcal{N}=1$ and a right $\tilde{\mathcal{N}}=0$ theories at linearized level: 
- Off-shell $\mathcal{N}=1$ Yang-Mills multiplet with $(4+4)$ bosonic + fermionic degrees of freedom and gauge group $G$ :

$$
A_{\mu}, \quad \psi, \quad D
$$

- Off-shell off-shell $\tilde{\mathcal{N}}=0$ Yang-Mills multiplet with $(3+0)$ bosonic + fermionic degrees of freedom and gauge group $\tilde{G}$ :

$$
\tilde{A}_{\nu}
$$

Without making any assumptions regarding the dynamics this yields the $(12+12)$ new-minimal $\mathcal{N}=1$ supergravity multiplet 45:

$$
g_{\mu \nu}, \quad B_{\mu \nu}, \quad \psi_{\mu}, \quad V_{\mu}
$$

where general covariance, 2 -form gauge invariance, local supersymmetry and local chiral symmetry follows from the left/right gauge symmetries.

The "gravity $=$ Yang-Mills $\times$ Yang-Mills" dictionary and symmetry transformations are most concisely expressed in the superfield formalism. Hence, we consider:

1. A left $\mathcal{N}=1$ real vector superfield,

$$
\begin{aligned}
V(x, \theta, \bar{\theta})= & C+i \theta \chi-i \bar{\theta} \bar{\chi}+i \theta^{2} F-i \bar{\theta}^{2} \bar{F}-\theta \sigma^{\mu} \bar{\theta} A_{\mu} \\
& +i \theta^{2} \bar{\theta}\left(\bar{\psi}+\frac{i}{2} \bar{\sigma}^{\rho} \partial_{\rho} \chi\right)-i \bar{\theta}^{2} \theta\left(\psi+\frac{i}{2} \sigma^{\rho} \partial_{\rho} \bar{\chi}\right) \\
& +\frac{1}{2} \bar{\theta}^{2} \theta^{2}\left(D+\frac{1}{2} \square C\right)
\end{aligned}
$$

transforming under local supergauge, non-Abelian global $G$ and global super-Poincaré:

$$
\delta V=\underbrace{\Lambda+\bar{\Lambda}}_{\text {local Abelian supergauge }}+\overbrace{[V, X]}^{\text {global non-Abelian } G}+\underbrace{\delta_{(a, \lambda, \epsilon)} V}_{\text {global super-Poincaré }}
$$

where $\Lambda(x, \theta, \bar{\theta})$ is a chiral superfield of supergauge parameters

$$
\Lambda(x, \theta, \bar{\theta})=B+\sqrt{2} \theta \zeta+\theta^{2} K+i \theta \sigma^{\rho} \bar{\theta} \partial_{\rho} a+\frac{i}{\sqrt{2}} \theta^{2} \bar{\theta} \bar{\sigma}^{\rho} \partial_{\rho} \zeta+\frac{1}{4} \theta^{2} \bar{\theta}^{2} \square B
$$

2. A right $\tilde{\mathcal{N}}=0$ Yang-Mills potential $\tilde{A}_{\nu}$ transforming under local gauge, non-Abelian global $\tilde{G}$ and global Poincaré:

$$
\delta \tilde{A}_{\nu}=\underbrace{\partial_{\nu} \tilde{\sigma}}_{\text {local Abelian gauge }}+\overbrace{\left[\tilde{A}_{\nu}, \tilde{X}\right]}^{\text {global non-Abelian } \tilde{G}}+\underbrace{\delta_{(a, \lambda)} A_{\nu}}_{\text {global Poincaré }}
$$

3. The spectator bi-adjoint scalar $\Phi$ field transforming under non-Abelian global $G \times \tilde{G}$ and global Poincaré:

$$
\delta \Phi=\overbrace{-[\Phi, X]-[\Phi, \tilde{X}]}^{\text {global non-Abelian } G \times \tilde{G}}+\underbrace{\delta_{a} \Phi}_{\text {global Poincaré }}
$$


The gravitational symmetries are reproduced here from those of Yang-Mills by invoking the gravity/YangMills dictionary for fields and supergauge parameters:

\begin{tabular}{|c|c|c|c|c|c|c|c|}
\hline Fields & $\varphi_{\nu}$ & $=$ & $V$ & $\star$ & $\Phi$ & $\star \tilde{A}_{\nu}$ & real superfield \\
\hline \multirow[t]{2}{*}{ Paras } & $\phi$ & $=$ & $V$ & $\star$ & $\Phi$ & $\star \tilde{\lambda}$ & real superfield \\
\hline & $S_{\nu}$ & $=$ & $\Lambda$ & $\star$ & $\Phi$ & $\star \tilde{A}_{\nu}$ & chiral superfield \\
\hline
\end{tabular}

Varying the gravitational superfield

$$
\begin{aligned}
\varphi_{\nu}(x, \theta, \bar{\theta})= & C_{\nu}+i \theta \chi_{\nu}-i \bar{\theta} \bar{\chi}_{\nu}+i \theta^{2} F_{\nu}-i \bar{\theta}^{2} \bar{F}_{\nu}-\theta \sigma^{\mu} \bar{\theta}\left(g_{\mu \nu}+B_{\mu \nu}\right) \\
& +i \theta^{2} \bar{\theta}\left(\bar{\psi}_{\nu}+\frac{i}{2} \bar{\sigma}^{\rho} \partial_{\rho} \chi_{\nu}\right)-i \bar{\theta}^{2} \theta\left(\psi_{\nu}+\frac{i}{2} \sigma^{\rho} \partial_{\rho} \bar{\chi}_{\nu}\right) \\
& +\frac{1}{2} \bar{\theta}^{2} \theta^{2}\left(V_{\nu}+\frac{1}{2} \square C_{\nu}\right)
\end{aligned}
$$

via the dictionary

$$
\delta \varphi_{\nu}=\delta V \star \Phi \star \tilde{A}_{\nu}+V \star \delta \Phi \star \tilde{A}_{\nu}+V \star \Phi \star \delta \tilde{A}_{\nu}
$$

we obtain

$$
\delta \varphi_{\nu}=S_{\nu}+\bar{S}_{\nu}+\partial_{\nu} \phi+\delta_{(a, \lambda, \epsilon)} \varphi_{\nu}
$$

This is the complete set of transformation rules for the new-minimal superfield at linearised approximation. Note, this derivation makes use of

$$
\langle[X, Y], Z\rangle=\langle X,[Y, Z]\rangle
$$

and, crucially, the convolution property

$$
\partial_{\mu}(f \star g)=\left(\partial_{\mu} f\right) \star g=f \star\left(\partial_{\mu} g\right) .
$$

To summarise, we have obtained the field content (4.9) and transformation rules (4.11) at linearised approximation of new-minimal $\mathcal{N}=1$ supergravity [46, 47]. Hence, the local gravitational symmetries of general covariance, 2-form gauge invariance, local supersymmetry and local chiral symmetry follow from those of Yang-Mills at linear level.

Introducing field equations we should match the on-shell content of the tensor product of spacetime little group representations. This is done covariantly by including the ghost sector in the dictionary [39, 40]. The $12+12$ multiplet splits with respect to superconformal transformations into an $8+8$ conformal supergravity multiplet plus a $4+4$ conformal tensor multiplet,

$$
\underbrace{\left(\begin{array}{c}
5+3+1+3 \\
4+2+4+2
\end{array}\right)}_{\text {new-minimal }} \rightarrow \underbrace{\left(\begin{array}{c}
5+3 \\
4+4
\end{array}\right)}_{\text {conformal }}+\underbrace{\left(\begin{array}{c}
3+1 \\
2+2
\end{array}\right)}_{\text {tensor }}
$$

in terms of $\mathrm{SO}(3)$ representions. Since the left (anti)ghost is a chiral superfield the ghost-antighost sector gives a compensating $4+4$ chiral (dilaton) multiplet [39,40], yielding old-minimal $12+12$ supergravity [48, 49] coupled to a tensor multiplet, which, with the conventional 2-derivative Lagrangian, correctly corresponds to the on-shell content obtained by tensoring left/right helicity states.

\section{$5 \quad$ Extended supersymmetry and U-duality}

This minimally supersymmetric example does not fully address the issue of U-duality [50], which, in context of string/M-theory, is of fundamental importance. U-duality manifests itself in supergravity, the 
low energy effective limit of string/M-theory, in the form of non-compact global symmetries, $\mathcal{G}$, acting non-linearly on the scalar fields [51. In all cases obtained from "Yang-Mills $\times$ Yang-Mills" the scalars parametrise a symmetric space $\mathcal{G} / \mathcal{H}$, where $\mathcal{H}$ is the maximal compact subgroup of $\mathcal{G}[52$. The Udualities and corresponding global symmetries for M-theory compactified on a $n$-torus are summarised in Table 1. Note, we will also use the term U-duality to refer to $\mathcal{G}$. The question of global symmetries from squaring Yang-Mills has also been addressed in [53 56], particularly in the context of scattering amplitudes.

\begin{tabular}{cccc}
\hline \hline$n$-torus & U-duality & $\mathcal{G}$ & $\mathcal{H}$ \\
\hline 1 & $\mathrm{SO}(1,1, \mathbb{Z})$ & $\mathrm{SO}(1,1, \mathbb{R})$ & - \\
2 & $\mathrm{SL}(2, \mathbb{Z}) \times \mathrm{SO}(1,1, \mathbb{Z})$ & $\mathrm{SL}(2, \mathbb{R}) \times \mathrm{SO}(1,1, \mathbb{R})$ & $\mathrm{SO}(2, \mathbb{R})$ \\
3 & $\mathrm{SL}(2, \mathbb{Z}) \times \mathrm{SL}(3, \mathbb{Z})$ & $\mathrm{SL}(2, \mathbb{R}) \times \mathrm{SL}(3, \mathbb{R})$ & $\mathrm{SO}(2, \mathbb{R}) \times \mathrm{SO}(3, \mathbb{R})$ \\
4 & $\mathrm{SL}(5, \mathbb{Z})$ & $\mathrm{SL}(5, \mathbb{R})$ & $\mathrm{SO}(5, \mathbb{R})$ \\
5 & $\mathrm{SO}(5,5, \mathbb{Z})$ & $\mathrm{SO}(5,5, \mathbb{R})$ & $\mathrm{SO}(5, \mathbb{R}) \times S O(5, \mathbb{R})$ \\
6 & $E_{6(6)}(\mathbb{Z})$ & $E_{6(6)}(\mathbb{R})$ & $\mathrm{USp}(8)$ \\
7 & $E_{7(7)}(\mathbb{Z})$ & $E_{7(7)}(\mathbb{R})$ & $\mathrm{SU}(8)$ \\
8 & $E_{8(8)}(\mathbb{Z})$ & $E_{8(8)}(\mathbb{R})$ & $\mathrm{SO}(16, \mathbb{R})$ \\
\hline \hline
\end{tabular}

Table 1: U-dualities (global symmetries) of M-theory $(D=11, \mathcal{N}=1$ supergravity) compactified on an $n$-torus.

As made clear by Table 1, U-duality becomes increasingly manifest as one descends in dimension $2^{2}$, Thus, to fully expose the structure of U-duality with respect to squaring we should consider the product in $D=3$ of left Yang-Mills theories with $\mathcal{N}=1,2,4,8$ and right Yang-Mills theories with $\tilde{\mathcal{N}}=1,2,4,8$. This was done in [61]. The result revealed a rather intriguing mathematical structure. The U-duality algebras obtained make up the Freudenthal-Rosenfeld-Tits magic square [62 64] as given in Table 2. As we shall explain this surprise has an elegant explanation, but first we must spend some time on the magic square itself.

Note, the real forms appearing in Table 2 are not unique; there are numerous possibilities as described in [65. They also play a role in supergravity. In particular, the $\mathbb{C}, \mathbb{H}$, and $\mathbb{O}$ rows of one such magic square (distinct from Table 2) describe the U-dualities of the aptly named magic supergravities in $D=5,4,3$ respectively $66[68]$. It should be emphasised, however, that the appearance of the magic square here is unrelated to these constructions.

\begin{tabular}{c|cccc}
\hline \hline $\mathcal{N} \otimes \tilde{N}$ & 1 & 2 & 4 & 8 \\
\hline & & & & \\
1 & $\mathfrak{s l}(2, \mathbb{R})$ & $\mathfrak{s u}(2,1)$ & $\mathfrak{s p}(4,2)$ & $\mathfrak{f}_{4(-20)}$ \\
2 & $\mathfrak{s u}(2,1)$ & $\mathfrak{s u}(2,1) \times \mathfrak{s u}(2,1)$ & $\mathfrak{s u}(4,2)$ & $\mathfrak{e}_{6(-14)}$ \\
4 & $\mathfrak{s p}(4,2)$ & $\mathfrak{s u}(4,2)$ & $\mathfrak{s o}(8,4)$ & $\mathfrak{e}_{7(-5)}$ \\
8 & $\mathfrak{f}_{4(-20)}$ & $\mathfrak{e}_{6(-14)}$ & $\mathfrak{e}_{7(-5)}$ & $\mathfrak{e}_{8(8)}$ \\
& & & & \\
\hline \hline
\end{tabular}

Table 2: The magic square of U-duality algebras obtained from the product of two Yang-Mills theories in $D=3$ spacetime dimensions.

\footnotetext{
${ }^{2}$ We stop at $D=3$, which has $E_{8(8)}$ U-duality, the largest finite dimensional exceptional Lie algebra. One can continue to $D=2,1,0$, invoking the infinite dimensional extended algebras $E_{9(9)}, E_{10(10)}, E_{11(11)}[57,60]$. Although we will not discuss theses cases here, it would be interesting to investigate whether they can be understood from the perspective of Yang-Mills squared.
} 


\subsection{Division algebras and the magic square}

In this section we follow closely [69,70]; we refer the reader to these works for more detailed explanations and proofs. An algebra $\mathbb{A}$ defined over $\mathbb{R}$ with identity element $e_{0}$, is said to be composition if it has a non-degenerate quadratic form ${ }^{3} \mathbf{n}: \mathbb{A} \rightarrow \mathbb{R}$ such that,

$$
\mathbf{n}(a b)=\mathbf{n}(a) \mathbf{n}(b), \quad \forall a, b \in \mathbb{A},
$$

where we denote the multiplicative product of the algebra by juxtaposition. Regarding $\mathbb{R} \subset \mathbb{A}$ as the scalar multiples of the identity $\mathbb{R} e_{0}$ we may decompose $\mathbb{A}$ into its "real" and "imaginary" parts $\mathbb{A}=\mathbb{R} \oplus \mathbb{A}^{\prime}$, where $\mathbb{A}^{\prime} \subset \mathbb{A}$ is the subspace orthogonal to $\mathbb{R}$. An arbitrary element $a \in \mathbb{A}$ may be written $a=\operatorname{Re}(a)+\operatorname{Im}(a)$. Here $\operatorname{Re}(a) \in \mathbb{R} e_{0}, \operatorname{Im}(a) \in \mathbb{A}^{\prime}$ and

$$
\operatorname{Re}(a)=\frac{1}{2}(a+\bar{a}), \quad \operatorname{Im}(a)=\frac{1}{2}(a-\bar{a}),
$$

where we have defined conjugation using the bilinear form,

$$
\bar{a}:=\left\langle a, e_{0}\right\rangle e_{0}-a, \quad\langle a, b\rangle:=\mathbf{n}(a+b)-\mathbf{n}(a)-\mathbf{n}(b) .
$$

A composition algebra $\mathbb{A}$ is said to be division if it contains no zero divisors,

$$
a b=0 \Rightarrow a=0 \quad \text { or } \quad b=0,
$$

in which case $\mathbf{n}$ is positive semi-definite and $\mathbb{A}$ is referred to as a normed division algebra. Hurwitz's celebrated theorem states that there are exactly four normed division algebras [71]: the reals, complexes, quaternions and octonions, denoted respectively by $\mathbb{R}, \mathbb{C}, \mathbb{H}$ and $\mathbb{O}$. They may be constructed via the Cayley-Dickson doubling procedure, $\mathbb{A}^{\prime}=\mathbb{A} \oplus \mathbb{A}$ with multiplication in $\mathbb{A}^{\prime}$ defined by

$$
(a, b)(c, d)=(a c-d \bar{b}, \bar{a} d+c b) .
$$

With each doubling a property is lost as summarised here:

$\begin{array}{lllllll}\mathbb{A} & \text { Construction } & \text { Dim } & \text { Division } & \text { Associative } & \text { Commutative } & \text { Ordered } \\ \mathbb{R} & \mathbb{R} & 1 & \text { yes } & \text { yes } & \text { yes } & \text { yes } \\ \mathbb{C} & \mathbb{R} \oplus \mathbb{R} & 2 & \text { yes } & \text { yes } & \text { yes } & \text { no } \\ \mathbb{H} & \mathbb{C} \oplus \mathbb{C} & 4 & \text { yes } & \text { yes } & \text { no } & \text { no } \\ \mathbb{O} & \mathbb{H} \oplus \mathbb{H} & 8 & \text { yes } & \text { no } & \text { no } & \text { no } \\ \mathbb{S} & \mathbb{O} \oplus \mathbb{O} & 16 & \text { no } & \text { no } & \text { no } & \text { no }\end{array}$

On doubling the octonions, $\mathbb{S} \cong \mathbb{O} \oplus \mathbb{O}$, the division property fails and we will not consider such cases here. Note that, while the octonions are not associative they are alternative:

$$
[a, b, c]:=(a b) c-a(b c)
$$

is an alternating function under the interchange of its arguments. This property is crucial for supersymmetry.

An element $a \in \mathbb{O}$ may be written $a=a^{a} e_{a}$, where $a=0, \ldots, 7, a^{a} \in \mathbb{R}$ and $\left\{e_{a}\right\}$ is a basis with one real $e_{0}$ and seven $e_{i}, i=1, \ldots, 7$, imaginary elements. The octonionic multiplication rule is,

$$
e_{a} e_{b}=\left(\delta_{a 0} \delta_{b c}+\delta_{0 b} \delta_{a c}-\delta_{a b} \delta_{0 c}+C_{a b c}\right) e_{c}
$$

where $C_{a b c}$ is totally antisymmetric and $C_{0 b c}=0$. The non-zero $C_{i j k}$ are given by the Fano plane. See Figure 2.

There are three symmetry algebras on $\mathbb{A}$ that we will make use of:

\footnotetext{
${ }^{3} \mathrm{~A}$ quadratic norm on a vector space $V$ over a field $\mathbb{R}$ is a map $\mathbf{n}: V \rightarrow \mathbb{R}$ such that: (1) $\mathbf{n}(\lambda a)=\lambda^{2} \mathbf{n}(a), \lambda \in \mathbb{R}, a \in V$ and $(2)\langle a, b\rangle:=\mathbf{n}(a+b)-\mathbf{n}(a)-\mathbf{n}(b)$ is bilinear.
} 


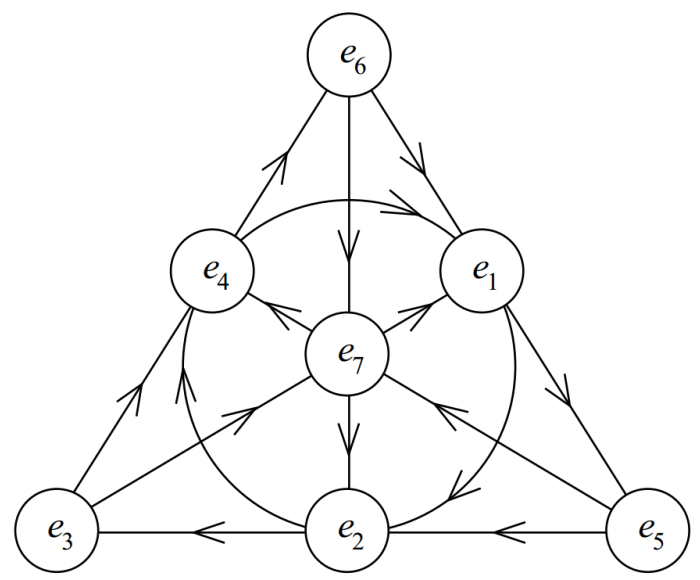

Figure 2: The Fano plane. The structure constants are determined by the Fano plane, $C_{i j k}=1$ if $i j k$ lies on a line and is ordered according as its orientation. Each oriented line follows the rules of quaternionic multiplication. For example, $e_{2} e_{3}=e_{5}$ and cyclic permutations; odd permutations go against the direction of the arrows on the Fano plane and we pick up a minus sign, e.g. $e_{3} e_{2}=-e_{5}$.

1. The norm preserving algebra is defined as,

$$
\mathfrak{s o}(\mathbb{A}):=\left\{A \in \operatorname{Hom}_{\mathbb{R}}(\mathbb{A}) \mid\langle A a, b\rangle+\langle a, A b\rangle=0, \forall a, b \in \mathbb{A}\right\}
$$

yielding,

$$
\begin{aligned}
& \mathfrak{s o}(\mathbb{R}) \cong \emptyset \\
& \mathfrak{s o}(\mathbb{C}) \cong \mathfrak{s o}(2), \\
& \mathfrak{s o}(\mathbb{H}) \cong \mathfrak{s o}(3) \oplus \mathfrak{s o}(3), \\
& \mathfrak{s o}(\mathbb{O}) \cong \mathfrak{s o}(8) .
\end{aligned}
$$

2. The triality algebra of $\mathbb{A}$ is defined as,

$$
\operatorname{tri}(\mathbb{A}):=\{(A, B, C) \in \mathfrak{s o}(\mathbb{A}) \oplus \mathfrak{s o}(\mathbb{A}) \oplus \mathfrak{s o}(\mathbb{A}) \mid A(a b)=B(a) b+a C(b), \forall a, b \in \mathbb{A}\},
$$

yielding,

$$
\begin{aligned}
\mathfrak{t r i}(\mathbb{R}) & \cong \emptyset \\
\mathfrak{t r i}(\mathbb{C}) & \cong \mathfrak{s o}(2) \oplus \mathfrak{s o}(2), \\
\mathfrak{t r i}(\mathbb{H}) & \cong \mathfrak{s o}(3) \oplus \mathfrak{s o}(3) \oplus \mathfrak{s o}(3), \\
\mathfrak{t r i}(\mathbb{O}) & \cong \mathfrak{s o}(8)
\end{aligned}
$$

3. One can regard the triality algebra as a generalised form of the derivation algebra defined as,

$$
\mathfrak{d e r}(\mathbb{A})=\left\{A \in \operatorname{Hom}_{\mathbb{R}}(\mathbb{A}) \mid A(a b)=A(a) b+a A(b)\right\},
$$

which for $\mathbb{A}=\mathbb{O}$ gives the smallest exceptional Lie algebra,

$$
\begin{aligned}
\mathfrak{d} \mathfrak{e r}(\mathbb{R}) & \cong \emptyset \\
\mathfrak{d e r}(\mathbb{C}) & \cong \emptyset \\
\mathfrak{d e r}(\mathbb{H}) & \cong \mathfrak{s o}(3), \\
\mathfrak{d e r}(\mathbb{O}) & \cong \mathfrak{g}_{2(-14)}
\end{aligned}
$$


This provides the first example of a division algebraic description of an exceptional Lie algebra. In fact, the entire Freudenthal magic square can be realised in terms of the division algebras. The magic square was the result of an effort to give a unified and geometrically motivated description of Lie algebras, including the remaining exceptional cases of $\mathfrak{f}_{4}, \mathfrak{e}_{6}, \mathfrak{e}_{7}, \mathfrak{e}_{8}$. The classical Lie algebras $\mathfrak{s o}(n), \mathfrak{s u}(2), \mathfrak{s p}(n)$ are very naturally captured by $\mathbb{R}, \mathbb{C}, \mathbb{H}$ geometrical structures, respectively. There are a number of ways of articulating this idea, but perhaps the most concise is in terms of the isometries of projective geometries:

$$
\mathfrak{I s o m}\left(\mathbb{R P}^{n}\right) \cong \mathfrak{s o}(n+1), \quad \mathfrak{I} \mathfrak{s o m}\left(\mathbb{C P}^{n}\right) \cong \mathfrak{s u}(n+1), \quad \mathfrak{I s o m}\left(\mathbb{H P}^{n}\right) \cong \mathfrak{s p}(n+1)
$$

This sequence is rather suggestive; we might expect octonionic projective geometries to yield exceptional Lie algebras. Despite non-associativity it was shown by Moufang [72 that one can consistently construct the octonionic projective line and plane, denoted $\mathrm{OP}^{1}$ and $\mathrm{OP}^{2}$, respectively. The latter is often referred to as the Cayley plane. We cannot go beyond $n=2$ for the octonions 4 , which in this context reflects the fact that there is indeed just a finite set of exceptional Lie algebras not belonging to any countably infinite family. The $\mathrm{OP}^{1}$ example is constructed in direct analogy with the real, complex and quaternionic cases5. It is diffeomorphic to $S^{8}$. The octonionic plane has a more intricate structure. An element $(a, b, c) \in \mathbb{O}^{3}$ with $\mathbf{n}(a)+\mathbf{n}(a)+\mathbf{n}(c)=1$ and $(a b) c=a(b c)$ gives a point in $\mathbb{O P}^{2}$ (the line through the origin containing $(a, b, c)$ in $\left.\mathbb{O}^{3}\right)$. It is not difficult to show the space of such elements is a 16-dimensional real manifold embedded in $\mathbb{O}^{3}$ through eight real constraints: $\mathbf{n}(a)+\mathbf{n}(a)+\mathbf{n}(c)=1$ and $(a b) c=a(b c)$. The lines in $\mathrm{OP}^{2}$ are copies of $\mathrm{OP}^{1}$ and there is a duality relation sending lines/points into points/lines preserving the incidence structure. Borel showed that $F_{4(-52)}$ is the isometry group of a 16-dimensional projective plane, which is none other than $\mathrm{OP}^{2}$. One can show that the points and lines in $\mathrm{OP}^{2}$ are in one-to-one incidence preserving correspondence with trace 1 and 2 projectors in the Jordan algebra of $3 \times 3$ octonionic Hermitian matrices $\mathfrak{J}_{3}(\mathbb{O})$ (treating projectors as propositions the incidence relation in $\mathfrak{J}_{3}^{\mathbb{O}}$ is given by implication) 73 . Then $F_{4(-52)}=\operatorname{Isom}\left(\mathbb{O P}^{2}\right)$ follows automatically from the result of Chevalley and Schafer that $F_{4(-52)}=\operatorname{Aut}\left(\mathfrak{J}_{3}(\mathbb{O})\right)$, the group preserving the Jordan product with Lie algebra $\mathfrak{d e r}\left(\mathfrak{J}_{3}(\mathbb{O})\right)$ [74. In summary, the sequence in $[5.13)$ is continued to include,

$$
\mathfrak{I s o m}\left(\mathbb{O P}^{2}\right) \cong \mathfrak{d e r}\left(\mathfrak{J}_{3}(\mathbb{O})\right) \cong \mathfrak{f}_{4(-52)} .
$$

Since $F_{4(-52)}$ acts transitively on the space of trace 1 projectors and the stabiliser of a given trace 1 projector is isomorphic to $\operatorname{Spin}(9)$ we have,

$$
\mathrm{OP}^{2} \cong F_{4(-52)} / \operatorname{Spin}(9) \text {. }
$$

The Cayley plane is a homogenous symmetric space with $T_{p}\left(\mathrm{OP}^{2}\right) \cong \mathbb{O}^{2}$, which carries the spinor representation of $\operatorname{Spin}(9)$; under $F_{4(-52)} \supset \operatorname{Spin}(9)$ we have

$$
52 \rightarrow 36+16
$$

or in a more division algebraic form,

$$
\begin{aligned}
\mathfrak{f}_{4(-52)} & \cong \mathfrak{s o}(9)+\mathbf{1 6} \\
& \cong \mathfrak{s o}(\mathbb{R} \oplus \mathbb{O})+\mathbb{O}^{2} \\
& \cong \mathfrak{s o}(\mathbb{O})+\mathbb{O}+\mathbb{O}+\mathbb{O}
\end{aligned}
$$

The three $\mathbb{O}$ terms in the final line transform in the three triality related 8-dimensional representations of $\mathfrak{s o}(8)$, the vector, spinor and conjugate spinor. It is this triality relation which implies that $\mathfrak{t r i}(\mathbb{O}) \cong \mathfrak{s o}(\mathbb{O})$.

\footnotetext{
${ }^{4}$ One way to understand this is in terms of Jordan algebras. Points in $\mathrm{OP}^{2}$ are bijectively identified with trace 1 projectors in $\mathfrak{J}_{3}^{\mathrm{O}}$, the Jordan algebra of $3 \times 3$ octonionic Hermitian matrices. However, for $m>3, m \times m$ octonionic Hermitian matrices do not form a Jordan algebra.

${ }^{5}$ Non-associativity, however, implies that the line through the origin containing the point $(a, b)$ is not given by $\{(\alpha a, \alpha b) \mid \alpha \in$ O \}, unless $x=1$ or $y=1$. This obstacle is easily avoided as all non-zero octonions have an inverse; $(a, b)$ is equivalent to $\left(b^{-1} a, 1\right)$ or $\left(1, a^{-1} b\right)$ for $b \neq 0$ or $a \neq 0$, giving two charts with a smooth transition function on their overlap. See 70 .
} 
Seemingly inspired by the trivial identity $\mathbb{O} \cong \mathbb{R} \otimes \mathbb{O}$ Boris Rosenfeld 64 proposed a natural extension of this construction,

$$
\mathfrak{I s o m}\left((\mathbb{C} \otimes \mathbb{O}) \mathbb{P}^{2}\right) \cong \mathfrak{e}_{6(-78)}, \quad \mathfrak{I s o m}\left((\mathbb{H} \otimes \mathbb{O}) \mathbb{P}^{2}\right) \cong \mathfrak{e}_{7(-133)}, \quad \mathfrak{I s o m}\left((\mathbb{O} \otimes \mathbb{O}) \mathbb{P}^{2}\right) \cong \mathfrak{e}_{8(-248)},
$$

thus giving a uniform geometric description for all Lie algebras. The would-be tangents spaces $(\mathbb{A} \otimes \mathbb{O})^{2}$ have the correct dimensions and representation theoretic properties. However, it is not actually possible to construct projective spaces over $\mathbb{H} \otimes \mathbb{O}$ and $\mathbb{O} \otimes \mathbb{O}$ using the logic applied to $\mathbb{O P}^{2}$, essentially because they do not yield Jordan algebras, unlike $\mathbb{C} \otimes \mathbb{O}$. They nonetheless can be identified with Riemannian geometries with isometries $E_{7(-133)}$ and $E_{8(-248)}$, respectively. Indeed, the Lie algebra decomposition $\varsigma^{6}$

$$
\begin{aligned}
\mathfrak{f}_{4(-52)} & \cong \mathfrak{s o}(\mathbb{R} \oplus \mathbb{O})+(\mathbb{R} \otimes \mathbb{O})^{2} \\
\mathfrak{e}_{6(-78)} & \cong \mathfrak{s o}(\mathbb{C} \oplus \mathbb{O}) \oplus \mathfrak{u}(1)+(\mathbb{C} \otimes \mathbb{O})^{2} \\
\mathfrak{e}_{7(-133)} & \cong \mathfrak{s o}(\mathbb{H} \oplus \mathbb{O}) \oplus \mathfrak{s p}(1)+(\mathbb{H} \otimes \mathbb{O})^{2} \\
\mathfrak{e}_{8(-248)} & \cong \mathfrak{s o}(\mathbb{O} \oplus \mathbb{O})+(\mathbb{O} \otimes \mathbb{O})^{2}
\end{aligned}
$$

naturally suggest the identifications

$$
\begin{aligned}
& \operatorname{Isom}\left((\mathbb{R} \otimes \mathbb{O}) \mathbb{P}^{2}\right)=F_{4(-52)} / \operatorname{Spin}(9) \\
& \operatorname{Isom}\left((\mathbb{C} \otimes \mathbb{O}) \mathbb{P}^{2}\right)=E_{6(-78)} /\left[(\operatorname{Spin}(10) \times \mathrm{U}(1)) / \mathbb{Z}_{4}\right] \\
& \operatorname{Isom}\left((\mathbb{H} \otimes \mathbb{O}) \mathbb{P}^{2}\right)=E_{7(-133)} /\left[(\operatorname{Spin}(10) \times \operatorname{Sp}(1)) / \mathbb{Z}_{2}\right] \\
& \operatorname{Isom}\left((\mathbb{O} \otimes \mathbb{O}) \mathbb{P}^{2}\right)=E_{8(-248)} /\left[\operatorname{Spin}(16) / \mathbb{Z}_{2}\right]
\end{aligned}
$$

with tangent spaces $(\mathbb{R} \otimes \mathbb{O})^{2},(\mathbb{C} \otimes \mathbb{O})^{2},(\mathbb{H} \otimes \mathbb{O})^{2},(\mathbb{O} \otimes \mathbb{O})^{2}$ carrying the appropriate spinor representations. Using the Tits' construction [63 the isometry algebras are given by the natural generalisation of (5.14),

$$
\begin{aligned}
\mathfrak{f}_{4(-52)} & \cong \mathfrak{d e r}(\mathbb{R}) \oplus \mathfrak{d e r}\left(\mathfrak{J}_{3}(\mathbb{O})\right)+\operatorname{ImR} \otimes \mathfrak{J}_{3}^{\prime}(\mathbb{O}) \\
\mathfrak{e}_{6(-78)} & \cong \mathfrak{d e r}(\mathbb{C}) \oplus \mathfrak{d e r}\left(\mathfrak{J}_{3}(\mathbb{O})\right)+\operatorname{ImC} \otimes \mathfrak{J}_{3}^{\prime}(\mathbb{O}) \\
\mathfrak{e}_{7(-133)} & \cong \mathfrak{d e r}(\mathbb{H}) \oplus \mathfrak{d e r}\left(\mathfrak{J}_{3}(\mathbb{O})\right)+\operatorname{ImH} \otimes \mathfrak{J}_{3}^{\prime}(\mathbb{O}) \\
\mathfrak{e}_{8(-248)} & \cong \mathfrak{d e r}(\mathbb{O}) \oplus \mathfrak{d e r}\left(\mathfrak{J}_{3}(\mathbb{O})\right)+\operatorname{ImO} \otimes \mathfrak{J}_{3}^{\prime}(\mathbb{O}),
\end{aligned}
$$

where $\mathfrak{J}^{\prime}$ denotes the subset of traceless elements in $\mathfrak{J}$. Generalising further, the Tits' construction defines a Lie algebra,

$$
\mathfrak{M}\left(\mathbb{A}_{1}, \mathbb{A}_{2}\right):=\operatorname{der}\left(\mathbb{A}_{1}\right) \oplus \mathfrak{d e r}\left(\mathfrak{J}_{3}\left(\mathbb{A}_{2}\right)\right)+\operatorname{Im} \mathbb{A}_{1} \otimes \mathfrak{J}_{3}^{\prime}\left(\mathbb{A}_{2}\right),
$$

for an arbitrary pair $\mathbb{A}_{1}, \mathbb{A}_{2}=\mathbb{R}, \mathbb{C}, \mathbb{H}, \mathbb{O}$, which yields the (compact) magic square given in Table 3 . The "magic" is that Table 3 symmetric about the diagonal despite the apparent asymmetry of (5.22). To obtain a magic square with the non-compact real forms that follow from squaring Yang-Mills, as given in Table 2, one can use a Lorentzian Jordan algebra 65],

$$
\mathfrak{M}^{\prime}\left(\mathbb{A}_{1}, \mathbb{A}_{2}\right):=\mathfrak{d e r}\left(\mathbb{A}_{1}\right) \oplus \mathfrak{d e r}\left(\mathfrak{J}_{1,2}\left(\mathbb{A}_{2}\right)\right)+\operatorname{Im} \mathbb{A}_{1} \otimes \mathfrak{J}_{1,2}^{\prime}\left(\mathbb{A}_{2}\right)
$$

Later we shall see that Yang-Mills squared gives an alternative form of (5.23), based on the BartonSudbery triality construction [69], that is manifestly symmetric in $\mathbb{A}_{1}, \mathbb{A}_{2}$ [61, 75]. This symmetric form reflects the fact that the squaring procedure is itself symmetric on interchanging the left and right theories.

\footnotetext{
${ }^{6}$ Note, the additional factors are given by intermediate algebras: $\mathfrak{t r i}(\mathbb{A}) / \mathfrak{i n t}(\mathbb{A})=\emptyset, \mathfrak{u}(1), \mathfrak{s p}(1), \emptyset$ for $\mathbb{A}=\mathbb{R}, \mathbb{C}, \mathbb{H}, \mathbb{O} 69$.
} 


\begin{tabular}{c|cccc}
\hline \hline$\otimes$ & $\mathbb{R}$ & $\mathbb{C}$ & $\mathbb{H}$ & $\mathbb{O}$ \\
\hline & & & & \\
$\mathbb{R}$ & $\mathfrak{s u}(2)$ & $\mathfrak{s u}(3)$ & $\mathfrak{s p}(6)$ & $\mathfrak{f}_{4(-52)}$ \\
$\mathbb{C}$ & $\mathfrak{s u}(3)$ & $\mathfrak{s u}(3) \times \mathfrak{s u}(3)$ & $\mathfrak{s u}(6)$ & $\mathfrak{e}_{6(-78)}$ \\
$\mathbb{H}$ & $\mathfrak{s p}(6)$ & $\mathfrak{s u}(6)$ & $\mathfrak{s o}(12)$ & $\mathfrak{e}_{7(-133)}$ \\
$\mathbb{O}$ & $\mathfrak{f}_{4(-52)}$ & $\mathfrak{e}_{6(-78)}$ & $\mathfrak{e}_{7(-133)}$ & $\mathfrak{e}_{8(-248)}$ \\
& & & & \\
\hline \hline
\end{tabular}

Table 3: The magic square given by the Tits' construction.

\subsection{Division algebras and Yang-Mills theories}

In the two previous sections we saw that the "square" of $D=3$ super Yang-Mills theories and the "square" of division algebras both led to the magic square of Freudenthal. Surely this is no coincidence. Indeed, there is a long history of work connecting supersymmetry, spacetime and the division algebras $[?, 65,68,70,76,104$, which as we shall review underlies this magical meeting.

Perhaps the most direct link from division algebras to spacetime symmetries comes via the Lie algebra isomorphism of Sudbery 81,

$$
\mathfrak{s l}(2, \mathbb{A}) \cong \mathfrak{s o}(1,1+\operatorname{dim} \mathbb{A})
$$

which identifies $D=3,4,6,10$ as algebraically special. This is itself tied to the earlier observation of Kugo and Townsend [80] that the existence of minimal super Yang-Mills mulitplets in only $D=3,4,6,10$ is related to the uniqueness of $\mathbb{R}, \mathbb{C}, \mathbb{H}, \mathbb{O}$. This was followed-up by a number of authors 105 109], sharpening the correspondence between supersymmetry and division algebras. The final case of $D=10, \mathbb{A}=\mathbb{O}$ was developed most carefully in 98, where the link between supersymmetry and the alternativity of $\mathbb{O}$ was emphasised.

Pulling together these ideas, it was shown in [110] that $\mathcal{N}$-extended super Yang-Mills theories in $D=n+2$ dimensions are completely specified (the field content, Lagrangian and transformation rules) by selecting an ordered pair of division algebras: $\mathbb{A}_{n}$ for the spacetime dimension and $\mathbb{A}_{n \mathcal{N}}$ for the degree of supersymmetry, where the subscripts denote the dimension of the algebras.

Consequently, the dual appearances of the magic square in $D=3$, or equivalently for $\mathbb{A}_{n}=\mathbb{R}$, can be explained by the observation that $D=3, \mathcal{N}=1,2,4,8$ Yang-Mills theories can be formulated with a single Lagrangian and a single set of transformation rules, using fields valued in $\mathbb{R}, \mathbb{C}, \mathbb{H}$ and $\mathbb{O}$, respectively 61. Tensoring an $\mathbb{A}$-valued $D=3$ super Yang-Mills multiplet with an $\tilde{\mathbb{A}}$-valued $D=3$ super Yang-Mills multiplet yields a $D=3$ supergravity mulitplet with fields valued in $\mathbb{A} \otimes \tilde{\mathbb{A}}$, making a magic square of U-dualities appear rather natural.

Let us now review in some more detail these constructions. The Lagrangian for $(n+2)$-dimensional $\mathcal{N}=1$ super Yang-Mills with gauge group $G$ over the division algebra $\mathbb{A}_{n}$ is given 110 by

$$
\mathcal{L}\left(\mathbb{A}_{n}\right)=-\frac{1}{4} F_{\mu \nu}^{A} F^{A \mu \nu}-\operatorname{Re}\left(i \lambda^{\dagger A} \bar{\sigma}^{\mu} D_{\mu} \lambda^{A}\right), \quad \lambda \in \mathbb{A}_{n}^{2},
$$

where the covariant derivative and field strength are given by the usual expressions

$$
\begin{aligned}
D_{\mu} \lambda^{A} & =\partial_{\mu} \lambda^{A}+g f_{B C}{ }^{A} A_{\mu}^{B} \lambda^{C}, \\
F_{\mu \nu}^{A} & =\partial_{\mu} A_{\nu}^{A}-\partial_{\nu} A_{\mu}^{A}+g f_{B C}{ }^{A} A_{\mu}^{B} A_{\nu}^{C},
\end{aligned}
$$

with $A=0, \ldots, \operatorname{dim}[G]$. The $\left\{\sigma^{\mu}\right\}$ are a basis for $\mathbb{A}_{n}$-valued Hermitian matrices - the straightforward generalisation of the usual complex Pauli matrices $90,91,110$ to all four normed division algebras, satisfying the usual Clifford algebra relations. We can use these to write the supersymmetry transformations:

$$
\delta A_{\mu}^{A}=\operatorname{Re}\left(i \lambda^{\dagger A} \bar{\sigma}_{\mu} \epsilon\right), \quad \delta \lambda^{A}=\frac{1}{4} F_{\mu \nu}^{A} \sigma^{\mu}\left(\bar{\sigma}^{\nu} \epsilon\right) .
$$


Note, since the octonions are non-associative the ordering of the parentheses is important. Moreover, the components $\lambda^{A a}$ are anti-commuting; we are dealing with the algebra of octonions defined over the Grassmanns and we cannot rely on the usual spinor identities to hold automatically. However, everything goes through, thanks principally to alternativity.

By dimensionally reducing these theories using the Dixon-halving techniques of [110], we arrive at the Lagrangian for super Yang-Mills in $D=n+2$ with $\mathcal{N}$ supersymmetries written over the division algebra $\mathbb{A}_{n \mathcal{N}}$. The division algebra associated with spacetime $\mathbb{A}_{n}$ is viewed as a subalgebra of $\mathbb{A}_{n \mathcal{N}}$. The resulting Lagrangian is:

$$
\begin{aligned}
\mathcal{L}\left(\mathbb{A}_{n}, \mathbb{A}_{n \mathcal{N}}\right)= & -\frac{1}{4} F_{\mu \nu}^{A} F^{A \mu \nu}-\frac{1}{2}\left\langle D_{\mu} \phi^{A} \mid D^{\mu} \phi^{A}\right\rangle-\operatorname{Re}\left(i \lambda^{\dagger A} \bar{\sigma}^{\mu} D_{\mu} \lambda^{A}\right) \\
& -g f_{B C}{ }^{A} \operatorname{Re}\left(i \lambda^{\dagger A} \varepsilon \phi^{B} \lambda^{C}\right)-\frac{1}{16} g^{2} f_{B C}{ }^{A} f_{D E}{ }^{A}\left\langle\phi^{B} \mid \phi^{D}\right\rangle\left\langle\phi^{C} \mid \phi^{E}\right\rangle,
\end{aligned}
$$

where $\lambda \in \mathbb{A}_{n \mathcal{N}}^{2}$ (so we have $\mathcal{N}$ spacetime spinors, each valued in $\mathbb{A}_{n}^{2}$ ) and $\phi$ is a scalar field taking values in $\phi \in \mathbb{A}_{n}^{\mathbb{C}}$, the subspace of $\mathbb{A}_{n \mathcal{N}}$ orthogonal to the $\mathbb{A}_{n}$ subalgebra. The $\left\{\bar{\sigma}^{\mu}\right\}$ are still a basis for $\mathbb{A}_{n}$-valued Hermitian matrices, again, with $\mathbb{A}_{n}$ viewed as a division subalgebra of $\mathbb{A}_{n \mathcal{N}}$. As noted in [110], the overall (spacetime little group plus internal) symmetry of the $\mathcal{N}=1$ theory in $D=n+2$ dimensions is given by the triality algebra, $\mathfrak{t r i}\left(\mathbb{A}_{n}\right)$. If we dimensionally reduce these theories we obtain super Yang-Mills with $\mathcal{N}$ supersymmetries whose overall symmetries are given by,

$$
\mathfrak{s y \mathfrak { m }}\left(\mathbb{A}_{n}, \mathbb{A}_{n \mathcal{N}}\right):=\left\{(A, B, C) \in \mathfrak{t r i}\left(\mathbb{A}_{n \mathcal{N}}\right) \mid\left[A, \mathfrak{s o}\left(\mathbb{A}_{n}\right)_{S T}\right]=0, \quad \forall A \notin \mathfrak{s o}\left(\mathbb{A}_{n}\right)_{S T}\right\},
$$

where $\mathfrak{s o}\left(\mathbb{A}_{n}\right)_{S T}$ is the subalgbra of $\mathfrak{s o}\left(\mathbb{A}_{n \mathcal{N}}\right)$ that acts as orthogonal transformations on $\mathbb{A}_{n} \subseteq \mathbb{A}_{n \mathcal{N}}$. The

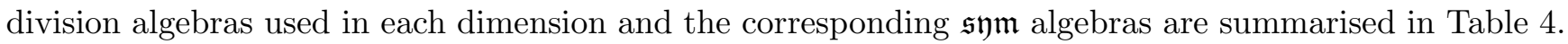

\begin{tabular}{c|cccc}
\hline \hline $\mathbb{A}_{n} \backslash \mathbb{A}_{n \mathcal{N}}$ & $\mathbb{O}$ & $\mathbb{H}$ & $\mathbb{C}$ & \\
\hline \multirow{O}{*}{} & $\mathfrak{s o}(8)_{S T}$ & & & \\
$\mathbb{H}$ & $\mathfrak{s o}(4)_{S T} \oplus \mathfrak{s p}(1) \oplus \mathfrak{s p}(1)$ & $\mathfrak{s o}(4)_{S T} \oplus \mathfrak{s p}(1)$ & & \\
$\mathbb{C}$ & $\mathfrak{s o}(2)_{S T} \oplus \mathfrak{s u}(4)$ & $\mathfrak{s o}(2)_{S T} \oplus \mathfrak{s p}(1) \oplus \mathfrak{s o}(2)$ & $\mathfrak{s o}(2)_{S T} \oplus \mathfrak{s o}(2)$ & \\
$\mathbb{R}$ & $\mathfrak{s o}(8)$ & $\mathfrak{s o}(4) \oplus \mathfrak{s p}(1)$ & $\mathfrak{s o}(2) \oplus \mathfrak{s o}(2)$ & $\emptyset$ \\
\hline \hline
\end{tabular}

Table 4: A table of algebras: $\mathfrak{s y m}\left(\mathbb{A}_{n}, \mathbb{A}_{n \mathcal{N}}\right)$. This lets us read off the spacetime and internal symmetries in each Yang-Mills theory. For example, one can see the familiar R-symmetries in $D=4$ : $\mathrm{U}(1), \mathrm{U}(2)$ and $\mathrm{SU}(4)$ for $\mathcal{N}=1,2,4$, respectively. Note that the symmetries in $D=3$ are entirely internal and that they include the R-symmetry as a subgroup (these are actually the symmetries of the theories after dualising the vector to a scalar).

Let us take $D=3$ as a concrete example. The $\mathcal{N}=8$ Lagrangian is given by

$$
\begin{aligned}
\mathcal{L}= & -\frac{1}{4} F_{\mu \nu}^{A} F^{A \mu \nu}-\frac{1}{2} D_{\mu} \phi_{i}^{A} D^{\mu} \phi_{i}^{A}+i \bar{\lambda}_{a}^{A} \gamma^{\mu} D_{\mu} \lambda_{a}^{A} \\
& -\frac{1}{4} g^{2} f_{B C}{ }^{A} f_{D E}{ }^{A} \phi_{i}^{B} \phi_{i}^{D} \phi_{j}^{C} \phi_{j}^{E} \\
& -g f_{B C}{ }^{A} \phi_{i}^{B} \bar{\lambda}^{A a} \Gamma_{a b}^{i} \lambda^{C b},
\end{aligned}
$$

where $\Gamma_{a b}^{i}, i=1, \ldots, 7, a, b=0, \ldots, 7$, belongs to the $\mathrm{SO}(7)$ Clifford algebra. The key observation is that this gamma matrix can be represented by the octonionic structure constants,

$$
\Gamma_{a b}^{i}=i\left(\delta_{b i} \delta_{a 0}-\delta_{b 0} \delta_{a i}+C_{i a b}\right)
$$


which allows us to rewrite the action over octonionic fields. If we replace $\mathbb{O}$ with a general division algebra $\mathbb{A}$, the result is $\mathcal{N}=1,2,4,8$ over $\mathbb{R}, \mathbb{C}, \mathbb{H}, \mathbb{O}$ :

$$
\begin{aligned}
\mathcal{L}= & -\frac{1}{4} F_{\mu \nu}^{A} F^{A \mu \nu}-\frac{1}{2} D_{\mu} \phi^{* A} D^{\mu} \phi^{A}+i \bar{\lambda}^{A} \gamma^{\mu} D_{\mu} \lambda^{A} \\
& -\frac{1}{4} g^{2} f_{B C}{ }^{A} f_{D E}{ }^{A}\left\langle\phi^{B} \mid \phi^{D}\right\rangle\left\langle\phi^{C} \mid \phi^{E}\right\rangle \\
& +\frac{i}{2} g f_{B C}{ }^{A}\left(\left(\bar{\lambda}^{A} \phi^{B}\right) \lambda^{C}-\bar{\lambda}^{A}\left(\phi^{* B} \lambda^{C}\right)\right)
\end{aligned}
$$

where $\phi=\phi^{i} e_{i}$ is an ImAl-valued scalar field, $\lambda=\lambda^{a} e_{a}$ is an $\mathbb{A}$-valued two-component spinor and $\bar{\lambda}=\bar{\lambda}^{a} e_{a}^{*}$.

The supersymmetry transformations in this language are given by

$$
\begin{aligned}
\delta \lambda^{A} & =\frac{1}{2}\left(F^{A \mu \nu}+\varepsilon^{\mu \nu \rho} D_{\rho} \phi^{A}\right) \sigma_{\mu \nu} \epsilon-\frac{1}{4} g f_{B C}{ }^{A} \phi^{B}\left(\phi^{C} \epsilon\right) \\
\delta A_{\mu}^{A} & =\frac{i}{2}\left(\bar{\epsilon} \gamma_{\mu} \lambda^{A}-\bar{\lambda}^{A} \gamma_{\mu} \epsilon\right) \\
\delta \phi^{A} & =\frac{i}{2} e_{i}\left[\left(\bar{\epsilon} e_{i}\right) \lambda^{A}-\bar{\lambda}^{A}\left(e_{i} \epsilon\right)\right]
\end{aligned}
$$

where $\epsilon$ is an $\mathbb{A}$-valued two-component spinor and $\sigma_{\mu \nu}$ are the generators of $\operatorname{SL}(2, \mathbb{R}) \cong \operatorname{Spin}(1,2)$. The form of the first term in the $\lambda^{A}$ transformation also highlights the vector's status as the missing real part of the $\operatorname{ImA}$-valued scalar field. Indeed, in the free $g=0$ theory one may dualise the vector to a scalar to obtain a full $\mathbb{A}$-valued field.

Now consider the product of two division algebraic multiplets:

1. A left $\mathcal{N}=\operatorname{dim} \mathbb{A}$ multiplet

$$
\left\{A_{\mu} \in \operatorname{Re} \mathbb{A}, \quad \phi \in \operatorname{Im} \mathbb{A}, \quad \lambda \in \mathbb{A}\right\}
$$

2. A right $\tilde{\mathcal{N}}=\operatorname{dim} \tilde{\mathbb{A}}$ multiplet

$$
\left\{\tilde{A}_{\nu} \in \operatorname{Re} \tilde{\mathbb{A}}, \quad \tilde{\phi} \in \operatorname{Im} \tilde{\mathbb{A}}, \quad \tilde{\lambda} \in \tilde{\mathbb{A}}\right\}
$$

We obtain the field content of an $(\mathcal{N}+\tilde{\mathcal{N}})$-extended supergravity theory valued in both $\tilde{\mathbb{A}}$ and $\tilde{\mathrm{A}}$ :

$$
g_{\mu \nu} \in \mathbb{R}, \quad \Psi_{\mu} \in\left(\begin{array}{l}
\mathbb{A} \\
\tilde{\mathbb{A}}
\end{array}\right), \quad \varphi, \chi \in\left(\begin{array}{c}
\mathbb{A} \otimes \tilde{\mathbb{A}} \\
\mathbb{A} \otimes \tilde{\mathbb{A}}
\end{array}\right) .
$$

The $\mathbb{R}$-valued graviton and $\mathbb{A} \oplus \tilde{\mathbb{A}}$-valued gravitino carry no degrees of freedom. The $(\mathbb{A} \otimes \tilde{\mathbb{A}})^{2}$-valued scalar and Majorana spinor each have $2(\operatorname{dim} \mathbb{A} \times \operatorname{dim} \tilde{\mathbb{A}})$ degrees of freedom.

The $\mathcal{H}$ algebra then follows immediately in this division algebraic language. The left and right factors each come with a commuting copy of the triality algebra, $\operatorname{tri}(\mathbb{A}) \oplus \operatorname{tri}(\tilde{\mathbb{A}})$. However, the $\mathbb{A} \otimes \tilde{\mathbb{A}} \operatorname{doublets}$ in (5.36) form irreducible representations of R-symmetry. The corresponding generators must themselves transform under $\mathfrak{t r i}(\mathbb{A}) \oplus \mathfrak{t r i}(\tilde{\mathbb{A}})$ consistently, implying they are elements of $\mathbb{A} \otimes \tilde{\mathbb{A}}$. This follows, formally, from the left/right supersymmetries. The conventional infinitesimal supersymmetry variation of the left $\otimes$ right states correctly gives the infinitesimal supersymmetry variation on the corresponding supergravity states [40,41,53. Seeking, instead, internal bosonic transformations on the supergavity multiplet suggests starting from the rather unconventional tensor product of the left and right supercharges, $Q \otimes \tilde{Q}$. See Figure 3. This follows, at least formally, from the observation $Q \otimes \tilde{Q} \in \mathbb{A} \otimes \tilde{\mathbb{A}}$, where we are explicitly suppressing the spacetime representation space. Note, these are "pseudo-supersymmetry" transformations since they do not change the mass dimension of the component fields. For an explicit construction see [52]. In summary, we have in total:

$$
\mathfrak{h}(\mathbb{A}, \tilde{\mathbb{A}}):=\mathfrak{t r i}(\mathbb{A}) \oplus \mathfrak{t r i}(\tilde{\mathbb{A}})+\mathbb{A} \otimes \tilde{\mathbb{A}}
$$




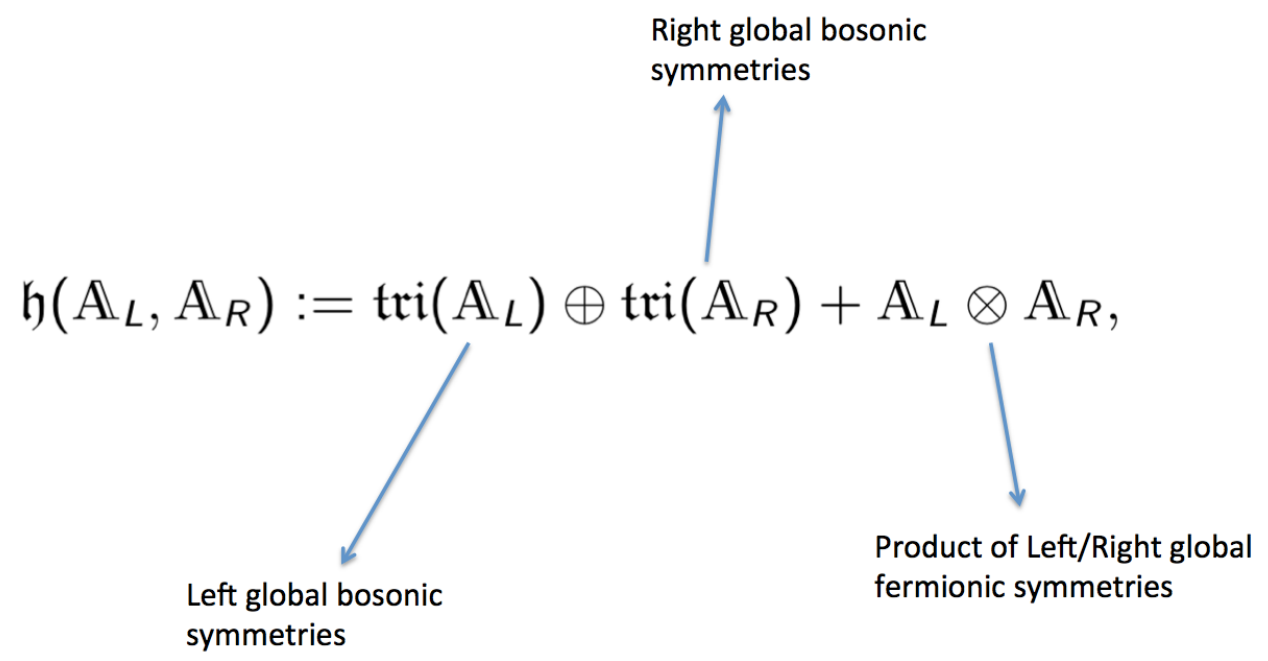

Figure 3: The $\mathcal{H}$ algebra in terms of the left/right super Yang-Mills theories in a division algebraic language.

\begin{tabular}{c|cccc}
\hline \hline $\mathbb{A}_{L} \backslash \mathbb{A}_{R}$ & $\mathbb{R}$ & $\mathbb{C}$ & $\mathbb{H}$ & $\mathbb{O}$ \\
\hline & & & & \\
$\mathbb{R}$ & $\mathfrak{s o}(2)$ & $\mathfrak{s o}(3) \times \mathfrak{s o}(2)$ & $\mathfrak{s o}(5) \times \mathfrak{s o}(3)$ & $\mathfrak{s o}(9)$ \\
$\mathbb{C}$ & $\mathfrak{s o}(3) \times \mathfrak{s o}(2)$ & {$[\mathfrak{s o}(3) \times \mathfrak{s o}(2)]^{2}$} & $\mathfrak{s o}(6) \times \mathfrak{s o}(3) \times \mathfrak{s o}(2)$ & $\mathfrak{s o}(10) \times \mathfrak{s o}(2)$ \\
$\mathbb{H}$ & $\mathfrak{s o}(5) \times \mathfrak{s o}(3)$ & $\mathfrak{s o}(6) \times \mathfrak{s o}(3) \times \mathfrak{s o}(2)$ & $\mathfrak{s o}(8) \times \mathfrak{s o}(4)$ & $\mathfrak{s o}(12) \times \mathfrak{s o}(3)$ \\
$\mathbb{O}$ & $\mathfrak{s o}(9)$ & $\mathfrak{s o}(10) \times \mathfrak{s o}(2)$ & $\mathfrak{s o}(12) \times \mathfrak{s o}(3)$ & $\mathfrak{s o}(16)$ \\
& & & & \\
\hline \hline
\end{tabular}

Table 5: Magic square of maximal compact subalgebras.

This Lie algebra, see 75 for the commutators, yields the maximal compact subalgebras of the corresponding U-dualities, given in Table 5 .

The U-dualities $\mathcal{G}$ are realised non-linearly on the scalars, which parametrise the symmetric spaces $\mathcal{G} / \mathcal{H}$. This can be understood using the identity relating $(\mathbb{A} \otimes \tilde{\mathbb{A}})^{2}$ to $\mathcal{G} / \mathcal{H}$,

$$
(\mathbb{A} \otimes \tilde{\mathbb{A}}) \mathbb{P}^{2} \cong \mathcal{G} / \mathcal{H}
$$

The scalar fields may be regarded as points in division-algebraic projective planes. The tangent space $T_{p}(\mathcal{G} / \mathcal{H}) \cong \mathfrak{p}=\mathfrak{g} \ominus \mathfrak{h}$ implies the scalars carry the $\mathfrak{p}$-representation of $\mathcal{H}$. The tangent space at any point of $(\mathbb{A} \otimes \tilde{\mathbb{A}}) \mathbb{P}^{2}$ is just $(\mathbb{A} \otimes \tilde{\mathbb{A}})^{2}$, the required representation space of $\mathcal{H}$. Since $\mathcal{G} / \mathcal{H}$ is a symmetric space, the U-duality Lie algebra is given by adjoining the scalar representation space $(\mathbb{A} \otimes \tilde{\mathbb{A}})^{2}$ to Figure 3 .

$$
\mathfrak{m}(\mathbb{A}, \tilde{\mathbb{A}}):=\underbrace{\operatorname{tri}(\mathbb{A}) \oplus \mathfrak{t r i}(\tilde{\mathbb{A}})+(\mathbb{A} \otimes \tilde{\mathbb{A}})}_{\mathfrak{h}(\mathbb{A}, \tilde{\mathbb{A}})}+\underbrace{(\mathbb{A} \otimes \tilde{\mathbb{A}})^{2}}_{\text {"scalars" }} .
$$

This has a $\mathbb{Z}_{2} \times \mathbb{Z}_{2}$ graded Lie algebra structure uniquely determined by the left/right super Yang-Mills factors and yields precisely the magic square. See 75 for a full account of the commutation relations. The triality construction described in $[69$ is isomorphic to $(5.39)$ as a vector space, but has a different Lie algebra structure, as reflected in the distinct real forms appearing in each case. In conclusion, the product of division algebras and super Yang-Mills theories both lead to the magic square, as depicted in Figure 4 . 


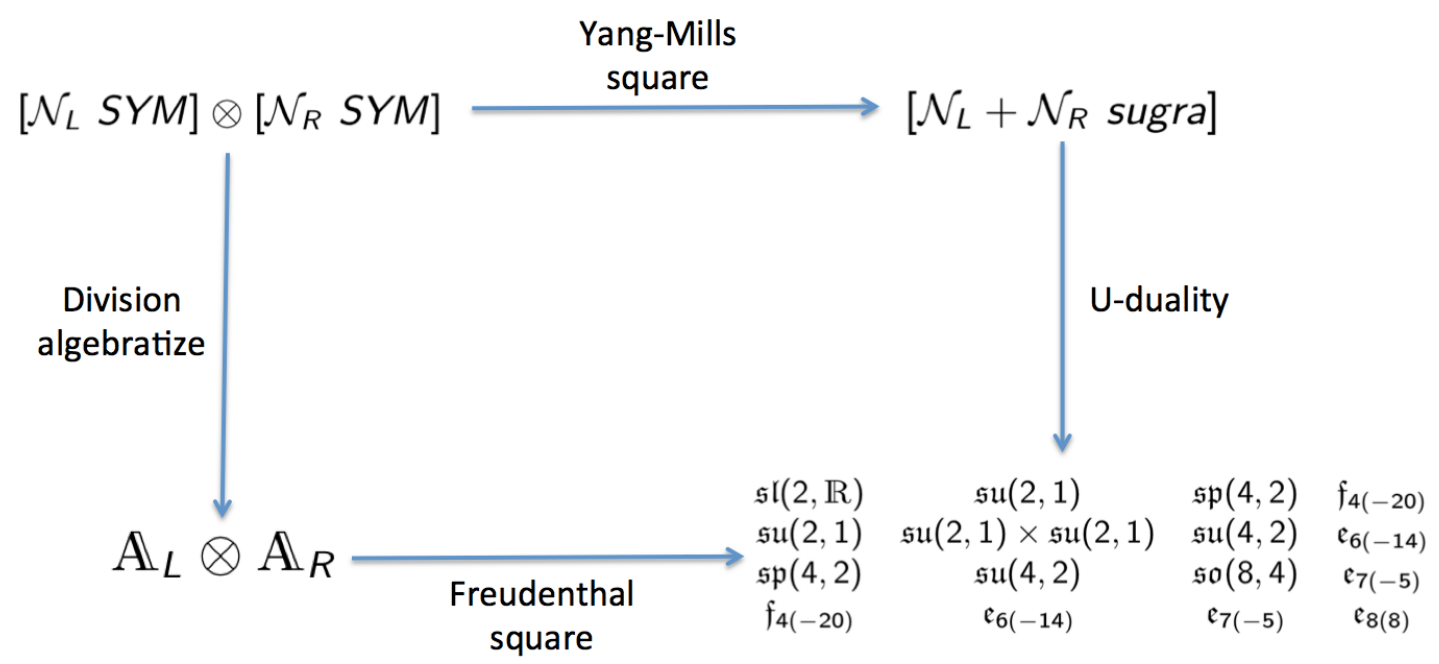

Figure 4: All roads lead to the magic square.

For $D=n+2$, we begin with a pair of Yang-Mills theories with $\mathcal{N}$ and $\tilde{\mathcal{N}}$ supersymmetries written over the division algebras $\mathbb{A}_{n \mathcal{N}}$ and $\mathbb{A}_{n \tilde{\mathcal{N}}}$, respectively, as described by (5.28). In terms of spacetime little group representations we may then write all the bosons of the left (right) theory as a single element $b \in \mathbb{A}_{n \mathcal{N}}\left(\tilde{b} \in \mathbb{A}_{n \tilde{\mathcal{N}}}\right)$, and similarly for the fermions $f \in \mathbb{A}_{n \mathcal{N}}\left(\tilde{f} \in \mathbb{A}_{n \tilde{\mathcal{N}}}\right)$. After tensoring we arrange the resulting supergravity fields into a bosonic doublet and a fermionic doublet,

$$
B=\left(\begin{array}{c}
b \otimes \tilde{b} \\
f \otimes \tilde{f}
\end{array}\right), \quad F=\left(\begin{array}{c}
b \otimes \tilde{f} \\
f \otimes \tilde{b}
\end{array}\right),
$$

just as we did in $D=3$. The algebra (5.37) acts naturally on these doublets. However, a diagonal $\mathfrak{s o}\left(\mathbb{A}_{n}\right)_{S T}$ subalgebra of this corresponds to spacetime transformations, so we must restrict $\mathfrak{h}\left(\mathbb{A}_{n \mathcal{N}}, \mathbb{A}_{n \tilde{\mathcal{N}}}\right)$ to the subalgebra that commutes with $\mathfrak{s o}\left(\mathbb{A}_{n}\right)_{S T}$. Heuristically, we identify a diagonal spacetime subalgebra $\mathbb{A}_{n}$ in $\mathbb{A}_{n \mathcal{N}} \otimes \mathbb{A}_{n \tilde{\mathcal{N}}}$ and require that it is preserved by the global isometries, which picks out a subset in $\mathfrak{I s o m}\left(\left(\mathbb{A}_{n \mathcal{N}} \otimes \mathbb{A}_{n \tilde{\mathcal{N}}}\right) \mathbb{P}^{2}\right)$. Imposing this condition selects the U-duality algebra of the $D=n+2$, $(\mathcal{N}+\tilde{\mathcal{N}})$-extended supergravity theory obtained by tensoring $\mathcal{N}$ and $\tilde{\mathcal{N}}$ super Yang-Mills theories. The Lie algebras are given by the magic pyramid formula:

$$
\mathfrak{M P} \mathfrak{y r}\left(\mathbb{A}_{n}, \mathbb{A}_{n \mathcal{N}}, \mathbb{A}_{n \tilde{\mathcal{N}}}\right):=\left\{u \in \mathfrak{m}\left(\mathbb{A}_{n \mathcal{N}}, \mathbb{A}_{n \tilde{\mathcal{N}}}\right)-\mathfrak{s o}\left(\mathbb{A}_{n}\right)_{S T} \mid\left[u, \mathfrak{s o}\left(\mathbb{A}_{n}\right)_{S T}\right]=0\right\} .
$$

The terminology is made clear by the pyramid of corresponding U-dualities groups presented in Figure 5 . The base of the pyramid in $D=3$ is the $4 \times 4$ Freudenthal magic square, while the higher levels are comprised of a $3 \times 3$ square in $D=4$, a $2 \times 2$ square in $D=6$ and Type II supergravity at the apex in $D=10$. Note, in [57 the oxidation of $\mathcal{N}$-extended $D=3$ dimensional supergravity theories was shown to generate a partially symmetric "trapezoid" of non-compact global symmetries for $D=3,4, \ldots 11$ and $0,2^{0}, 2^{1}, \ldots 2^{7}$ supercharges. A subset of algebras in the trapezoid with $D=3,4,5$ and $2^{5}, 2^{6}, 2^{7}$ supercharges matches the $D=3,4,5$ and $\mathbb{A}=\mathbb{C}, \mathbb{H}, \mathbb{O}$ exterior wall of the pyramid of Figure 5 .

Let us conclude with some comments on the product of theories other than super Yang-Mills. Particularly interesting examples are provided by the superconformal multiplets in $D=3,4,6$. In a manner directly analogous to the magic pyramid the tensor product of left and right superconformal theories yields the "conformal pyramid", described in 75]. It has the remarkable property that its faces are also given by the Freudenthal magic square, as depicted in Figure 6. In particular, ascending up the maximal spine one encounters the famous exceptional sequence $E_{8(8)}, E_{7(7)}, E_{6(6)}$, but where $E_{6(6)}$ belongs to the $D=6,(4,0)$ theory proposed by Hull as the superconformal limit of M-theory compactified on a 6-torus 111 113]. This pattern suggests the existence of some highly exotic $D=10$ theory with $F_{4(4)}$ 
O

$\mathrm{O}(1,1)$

O

H

$\mathrm{SU}^{*}(4) \times \mathrm{USp}(2) \mathrm{O}_{1} \mathrm{O}(4) \times \mathrm{O}(1,1)$

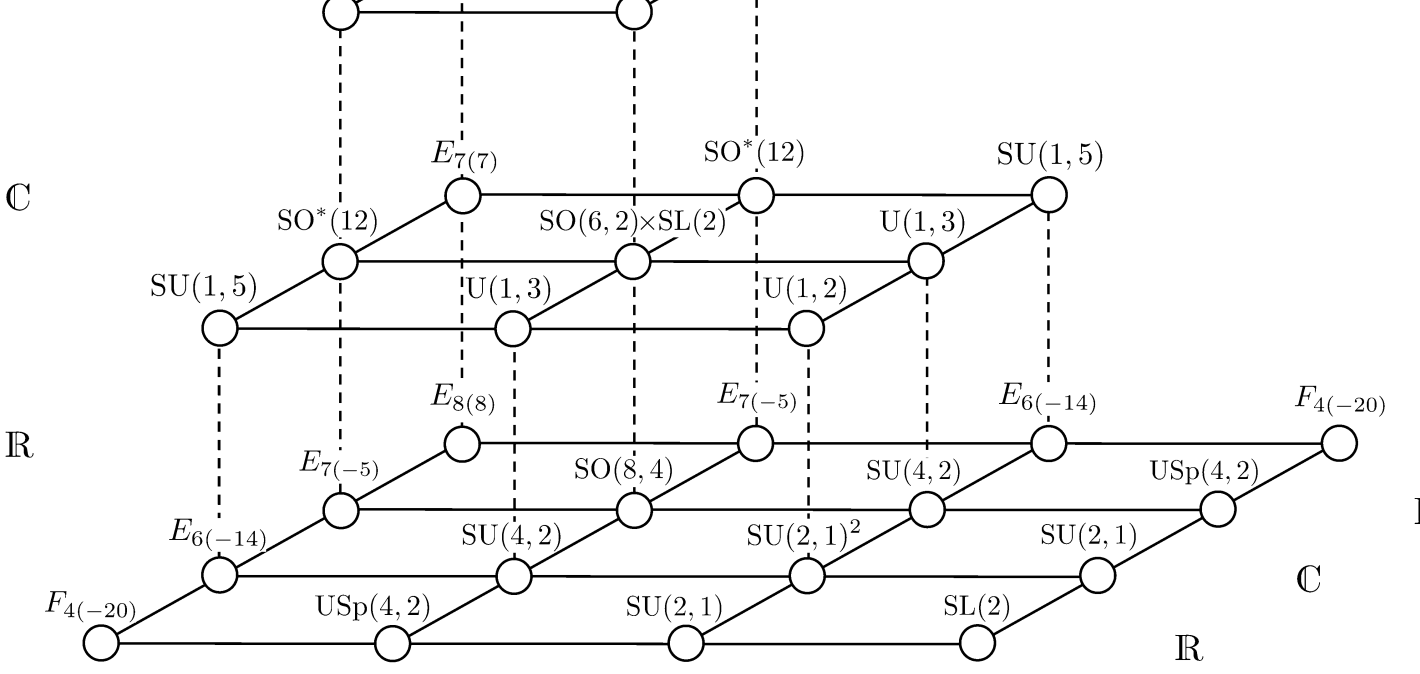

$\mathbb{O}$

$\mathrm{H}$

$\mathbb{C}$

$\mathbb{R}$

Figure 5: A magic pyramid of supergravities. The vertical axis labels the spacetime division algebra $\mathbb{A}_{n}$, while the horizontal axes label the algebras associated with the number of supersymmetries $\mathbb{A}_{n \mathcal{N}}$ and $\mathbb{A}_{n \tilde{\mathcal{N}}}$. 


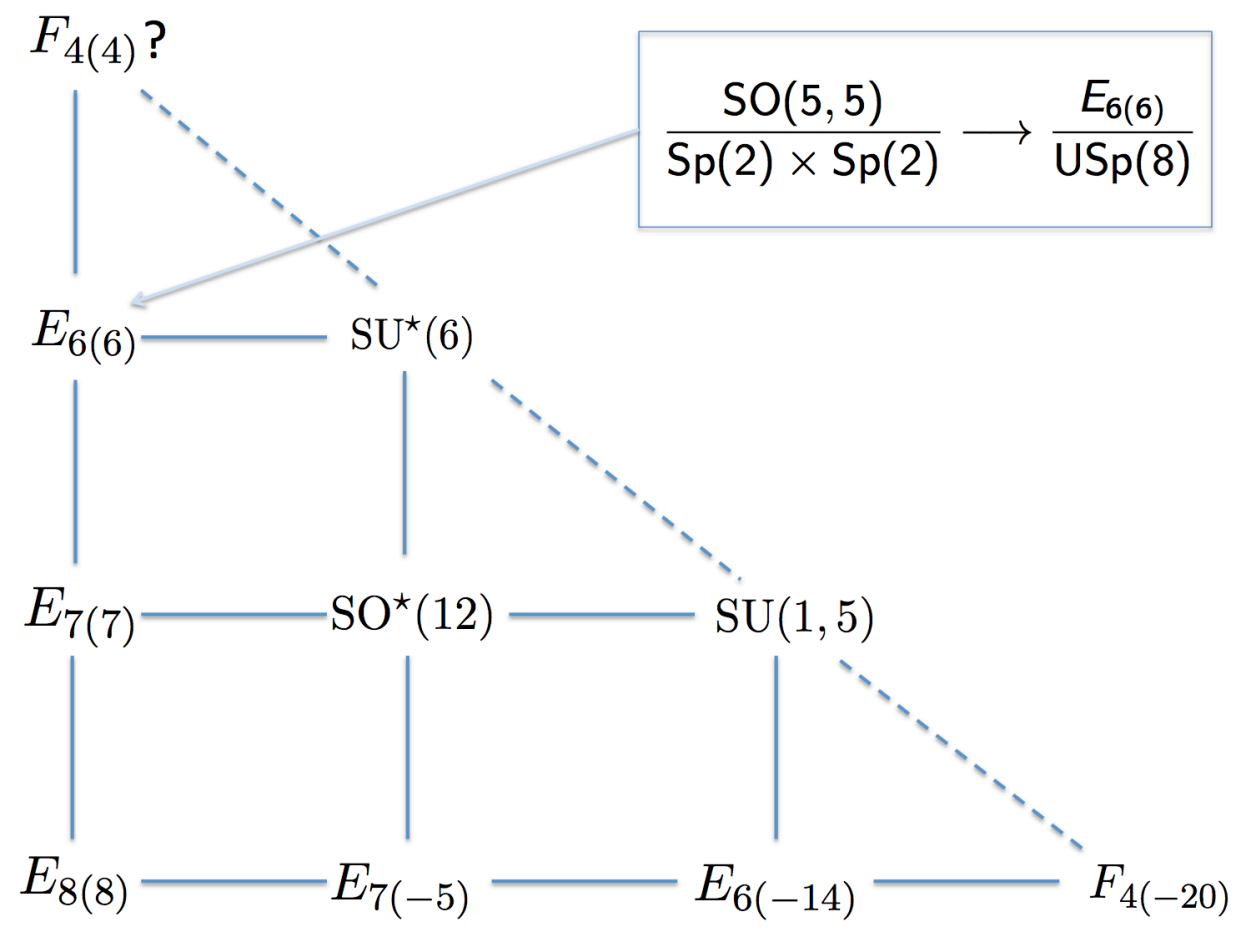

Figure 6: The magic faces of the conformal pyramid. We have highlighted the the maxiamally supersymmetric case in $D=6$. The $\mathcal{N}=(2,2)$ supergravity theory of the magic pyramid with U-Duality $\operatorname{SO}(5,5)$ is replaced by the $\mathcal{N}=(4,0)$ superconfromal theory, proposed by Hull [111 113], with U-duality $E_{6(6)}$. As a consequence, the outer faces of the conformal pyramid are diagonal slices of the Freudenthal magic square, upto the $F_{4(4)}$ slot, which currently has does not have a theory associated to it.

U-duality group. The existence of such a theory would be more than a little surprising and there is a (slightly) more conventional interpretation of the conformal pyramid, including its $F_{4(4)}$ tip, but for theories in $D=3,4,5,6$, as described 75$]$.

The product of conformal theories in the context of amplitudes has been considered previously in, for example, 54, 114 117]. In particular, the maximally supersymmetric $D=3, \mathcal{N}=8$ Bagger-LambertGustavsson (BLG) Chern-Simons-matter theory 118 120 has been shown to enjoy a colour-kinematic duality reflecting its three-algebra structure [116]. The "square" of BLG amplitudes yields those of $\mathcal{N}=16$ supergravity. Since $\mathcal{N}=16$ supergravity is the unique theory with 32 supercharges in three dimensions it is also the "square" of the $\mathcal{N}=8$ Yang-Mills theory. The square of the amplitudes in both cases agree, despite their distinct structures $[117$.

In $D=6$ one might expect relations between the "square" of the $\mathcal{N}=(2,0)$ tensor multiplet and the $\mathcal{N}=(4,0)$ theory proposed by Hull $[111-113]$, as discussed in [54]. Of course, amplitudes are generically not well-defined in these cases, but one can make some precise statements in terms of the tree-level $S$-matrix in particular regimes, as discussed in [114, 115]. For example, in the absence of additional degrees of freedom all tree-level amplitudes of the $(2,0)$ tensor multiplet vanish [114]. The $D=5, \mathcal{N}=4$ super Yang-Mills theory squares to give the amplitudes of $D=5, \mathcal{N}=8$ supergravity. However, being non-renormalisable it ought to be regarded as a superconformal $D=6, \mathcal{N}=(2,0)$ theory compactified on a circle of radius $R=g_{Y M}^{2} / 4 \pi^{2}$. At linearised level Hull's $(4,0)$ theory follows from the square the $(2,0)$ theory [121] and gives $\mathcal{N}=8, D=5$ supergravity when compactified on a circle [112. From this perspective the $(2,0) \times(2,0)=(4,0)$ identity constitutes an, as yet ill-defined, M-theory up-lift of the maximally supersymmetric $D=5$ squaring relation. 


\section{Acknowledgments}

MJD is grateful to the organisers of the Arnowitt Memorial Symposium for the invitation to contribute. Based on work done in collaboration with A. Anastasiou, M. J. Hughes and S. Nagy. We are very grateful for their numerous and essential contributions. We would also like to thank A. Marrani for his insights on the symmetries of supergravity. The work of LB was supported by a Schödinger Fellowship. The work of MJD is supported by the STFC under rolling grant ST/G000743/1.

\section{References}

[1] R. Utiyama, "Invariant Theoretical Interpretation of Interaction," Physical Review 101 (Mar., 1956) 1597-1607.

[2] T. W. B. Kibble, "Lorentz Invariance and the Gravitational Field," Journal of Mathematical Physics 2 (Mar., 1961) 212-221.

[3] S. W. MacDowell and F. Mansouri, "Unified geometric theory of gravity and supergravity," Physical Review Letters 38 (Apr., 1977) 739-742.

[4] A. H. Chamseddine and P. C. West, "Supergravity as a gauge theory of supersymmetry," Nuclear Physics B 129 (Oct., 1977) 39-44.

[5] K. S. Stelle and P. C. West, "de Sitter gauge invariance and the geometry of the Einstein-Cartan theory," Journal of Physics A Mathematical General 12 (Aug., 1979) L205-L210.

[6] G. Hooft, "Dimensional reduction in quantum gravity," Salamfestschrift: a collection of talks 4 (1994) no. A, 1-13, gr-qc/9310026.

[7] L. Susskind, "The World as a hologram," J.Math.Phys. 36 (1995) 6377-6396, arXiv:hep-th/9409089 [hep-th].

[8] J. M. Maldacena, "The large N limit of superconformal field theories and supergravity," Adv. Theor. Math. Phys. 2 (1998) 231-252, arXiv:hep-th/9711200.

[9] E. Witten, "Anti-de Sitter space and holography," Adv.Theor.Math.Phys. 2 (1998) 253-291, arXiv:hep-th/9802150 [hep-th].

[10] S. Gubser, I. R. Klebanov, and A. M. Polyakov, "Gauge theory correlators from noncritical string theory," Phys.Lett. B428 (1998) 105-114, arXiv:hep-th/9802109 [hep-th].

[11] H. Kawai, D. Lewellen, and S. Tye, "A Relation Between Tree Amplitudes of Closed and Open Strings," Nucl.Phys. B269 (1986) 1.

[12] Z. Bern, J. Carrasco, and H. Johansson, "New Relations for Gauge-Theory Amplitudes," Phys.Rev. D78 (2008) 085011, arXiv:0805.3993 [hep-ph].

[13] Z. Bern, J. J. M. Carrasco, and H. Johansson, "Perturbative Quantum Gravity as a Double Copy of Gauge Theory," Phys.Rev.Lett. 105 (2010) 061602, arXiv:1004.0476 [hep-th].

[14] H. Elvang and Y.-t. Huang, "Scattering Amplitudes," arXiv:1308.1697 [hep-th].

[15] Z. Bern, T. Dennen, Y.-t. Huang, and M. Kiermaier, "Gravity as the Square of Gauge Theory," Phys.Rev. D82 (2010) 065003, arXiv:1004.0693 [hep-th].

[16] J. J. M. Carrasco, "Gauge and Gravity Amplitude Relations," arXiv:1506.00974 [hep-th]. 
[17] Z. Bern, J. J. Carrasco, L. J. Dixon, H. Johansson, and R. Roiban, "The Ultraviolet Behavior of N=8 Supergravity at Four Loops," Phys. Rev. Lett. 103 (2009) 081301, arXiv:0905.2326 [hep-th].

[18] Z. Bern, S. Davies, and T. Dennen, "Enhanced ultraviolet cancellations in $\mathcal{N}=5$ supergravity at four loops," Phys.Rev. D90 (2014) no. 10, 105011, arXiv:1409.3089 [hep-th].

[19] R. Monteiro and D. O'Connell, "The Kinematic Algebra From the Self-Dual Sector," JHEP 1107 (2011) 007, arXiv:1105.2565 [hep-th].

[20] R. Monteiro and D. O'Connell, "The Kinematic Algebras from the Scattering Equations," JHEP 1403 (2014) 110, arXiv:1311.1151 [hep-th].

[21] N. E. J. Bjerrum-Bohr, P. H. Damgaard, and P. Vanhove, "Minimal Basis for Gauge Theory Amplitudes," Phys. Rev. Lett. 103 (2009) 161602, arXiv:0907.1425 [hep-th].

[22] S. Stieberger, "Open \& Closed vs. Pure Open String Disk Amplitudes," arXiv:0907.2211 [hep-th].

[23] S. H. Henry Tye and Y. Zhang, "Dual Identities inside the Gluon and the Graviton Scattering Amplitudes," JHEP 06 (2010) 071, arXiv: 1003.1732 [hep-th]. [Erratum: JHEP04,114(2011)].

[24] C. R. Mafra, O. Schlotterer, and S. Stieberger, "Explicit BCJ Numerators from Pure Spinors," JHEP 07 (2011) 092, arXiv: 1104.5224 [hep-th].

[25] C. R. Mafra and O. Schlotterer, "Berends-Giele recursions and the BCJ duality in superspace and components," arXiv:1510.08846 [hep-th].

[26] C. R. Mafra and O. Schlotterer, "Towards one-loop SYM amplitudes from the pure spinor BRST cohomology," Fortsch. Phys. 63 (2015) no. 2, 105-131, arXiv:1410.0668 [hep-th].

[27] C. R. Mafra and O. Schlotterer, "Two-loop five-point amplitudes of super Yang-Mills and supergravity in pure spinor superspace," JHEP 10 (2015) 124, arXiv:1505.02746 [hep-th].

[28] S. He, R. Monteiro, and O. Schlotterer, "String-inspired BCJ numerators for one-loop MHV amplitudes," JHEP 01 (2016) 171, arXiv:1507.06288 [hep-th].

[29] P. S. Howe and K. Stelle, "The Ultraviolet Properties of Supersymmetric Field Theories," Int.J.Mod.Phys. A4 (1989) 1871.

[30] S. Deser, J. H. Kay, and K. S. Stelle, "Renormalizability Properties of Supergravity," Phys. Rev. Lett. 38 (1977) 527, arXiv: 1506.03757 [hep-th].

[31] M. B. Green, J. G. Russo, and P. Vanhove, "String theory dualities and supergravity divergences," JHEP 1006 (2010) 075, arXiv:1002.3805 [hep-th].

[32] G. Bossard, P. Howe, and K. Stelle, "On duality symmetries of supergravity invariants," JHEP 1101 (2011) 020, arXiv: 1009.0743 [hep-th].

[33] N. Beisert, H. Elvang, D. Z. Freedman, M. Kiermaier, A. Morales, et al., "E7(7) constraints on counterterms in N=8 supergravity," Phys.Lett. B694 (2010) 265-271, arXiv:1009.1643 [hep-th].

[34] G. Bossard, P. Howe, K. Stelle, and P. Vanhove, "The vanishing volume of D=4 superspace," Class.Quant.Grav. 28 (2011) 215005, arXiv:1105.6087 [hep-th]. 
[35] G. Bossard, P. S. Howe, and K. S. Stelle, "Anomalies and divergences in N=4 supergravity," Phys. Lett. B719 (2013) 424-429, arXiv:1212.0841 [hep-th].

[36] R. Monteiro, D. O'Connell, and C. D. White, "Black holes and the double copy," JHEP 1412 (2014) 056, arXiv:1410.0239 [hep-th].

[37] A. Luna, R. Monteiro, D. O'Connell, and C. D. White, "The classical double copy for Taub-NUT spacetime," Phys. Lett. B750 (2015) 272-277, arXiv:1507.01869 [hep-th].

[38] A. Luna, R. Monteiro, I. Nicholson, D. O'Connell, and C. D. White, "The double copy: Bremsstrahlung and accelerating black holes," JHEP 06 (2016) 023, arXiv:1603.05737 [hep-th].

[39] W. Siegel, "SUPERSTRINGS GIVE OLD MINIMAL SUPERGRAVITY," Phys.Lett. B211 (1988) 55 .

[40] W. Siegel, "Curved extended superspace from Yang-Mills theory a la strings," Phys.Rev. D53 (1996) 3324-3336, arXiv:hep-th/9510150 [hep-th].

[41] A. Anastasiou, L. Borsten, M. J. Duff, L. J. Hughes, and S. Nagy, "Yang-Mills origin of gravitational symmetries," Phys.Rev.Lett. 113 (2014) no. 23, 231606, arXiv:1408.4434 [hep-th].

[42] A. Hodges, "New expressions for gravitational scattering amplitudes," Journal of High Energy Physics 1307 (2013), arXiv:1108.2227 [hep-th].

[43] F. Cachazo, S. He, and E. Y. Yuan, "Scattering of Massless Particles: Scalars, Gluons and Gravitons," JHEP 1407 (2014) 033, arXiv:1309.0885 [hep-th].

[44] L. Dolan and P. Goddard, "Proof of the Formula of Cachazo, He and Yuan for Yang-Mills Tree Amplitudes in Arbitrary Dimension," JHEP 1405 (2014) 010, arXiv:1311.5200 [hep-th].

[45] M. F. Sohnius and P. C. West, "An Alternative Minimal Off-Shell Version of N=1 Supergravity," Phys.Lett. B105 (1981) 353.

[46] S. Cecotti, S. Ferrara, M. Porrati, and S. Sabharwal, "NEW MINIMAL HIGHER DERIVATIVE SUPERGRAVITY COUPLED TO MATTER," Nucl.Phys. B306 (1988) 160.

[47] S. Ferrara and S. Sabharwal, "Structure of New Minimal Supergravity," Annals Phys. 189 (1989) 318-351.

[48] K. Stelle and P. C. West, "Minimal Auxiliary Fields for Supergravity," Phys.Lett. B74 (1978) 330.

[49] S. Ferrara and P. van Nieuwenhuizen, "The Auxiliary Fields of Supergravity," Phys.Lett. B74 (1978) 333

[50] C. M. Hull and P. K. Townsend, "Unity of superstring dualities," Nucl. Phys. B438 (1995) 109-137, arXiv:hep-th/9410167.

[51] E. Cremmer and B. Julia, "The SO(8) supergravity," Nucl. Phys. B159 (1979) 141.

[52] A. Anastasiou, L. Borsten, L. J. Hughes, and S. Nagy, "Global symmetries of Yang-Mills squared in various dimensions," JHEP 148 (2016) 1601, arXiv:1502.05359 [hep-th].

[53] M. Bianchi, H. Elvang, and D. Z. Freedman, "Generating Tree Amplitudes in N=4 SYM and N = 8 SG," JHEP 0809 (2008) 063, arXiv:0805.0757 [hep-th]. 
[54] M. Chiodaroli, M. Gunaydin, and R. Roiban, "Superconformal symmetry and maximal supergravity in various dimensions," JHEP 1203 (2012) 093, arXiv:1108.3085 [hep-th].

[55] J. J. M. Carrasco, M. Chiodaroli, M. Günaydin, and R. Roiban, "One-loop four-point amplitudes in pure and matter-coupled N = 4 supergravity," JHEP 1303 (2013) 056, arXiv:1212.1146 [hep-th].

[56] M. Chiodaroli, M. Günaydin, H. Johansson, and R. Roiban, "Scattering amplitudes in $\mathcal{N}=2$ Maxwell-Einstein and Yang-Mills/Einstein supergravity," JHEP 01 (2015) 081, arXiv:1408.0764 [hep-th].

[57] B. Julia, "Group disintegrations," in Superspace and Supergravity, S. Hawking and M. Rocek, eds., vol. C8006162 of Nuffield Gravity Workshop, pp. 331-350. Cambridge University Press, 1980.

[58] H. Nicolai, "The Integrability of $N=16$ Supergravity," Phys.Lett. B194 (1987) 402.

[59] P. C. West, "E(11) and M theory," Class.Quant.Grav. 18 (2001) 4443-4460, arXiv:hep-th/0104081 [hep-th].

[60] T. Damour, M. Henneaux, and H. Nicolai, "E(10) and a 'small tension expansion' of M theory," Phys.Rev.Lett. 89 (2002) 221601, arXiv:hep-th/0207267 [hep-th].

[61] L. Borsten, M. J. Duff, L. J. Hughes, and S. Nagy, "A magic square from Yang-Mills squared," Phys.Rev.Lett. 112 (2014) 131601, arXiv:1301.4176 [hep-th].

[62] H. Freudenthal, "Beziehungen der $E_{7}$ und $E_{8}$ zur oktavenebene I-II," Nederl. Akad. Wetensch. Proc. Ser. 57 (1954) 218-230.

[63] J. Tits, "Interprétation géométriques de groupes de Lie simples compacts de la classe E," Mém. Acad. Roy. Belg. Sci 29 (1955) 3.

[64] B. A. Rosenfeld, "Geometrical interpretation of the compact simple Lie groups of the class $E$ (Russian)," Dokl. Akad. Nauk. SSSR 106 (1956) 600-603.

[65] S. L. Cacciatori, B. L. Cerchiai, and A. Marrani, "Squaring the Magic," arXiv:1208.6153 [math-ph]

[66] M. Günaydin, G. Sierra, and P. K. Townsend, "Exceptional supergravity theories and the magic square," Phys. Lett. B133 (1983) 72.

[67] M. Günaydin, G. Sierra, and P. K. Townsend, "The geometry of $N=2$ Maxwell-Einstein supergravity and Jordan algebras," Nucl. Phys. B242 (1984) 244.

[68] M. Günaydin, G. Sierra, and P. K. Townsend, "Gauging the $d=5$ Maxwell-Einstein supergravity theories: More on Jordan algebras," Nucl. Phys. B253 (1985) 573.

[69] C. H. Barton and A. Sudbery, "Magic squares and matrix models of Lie algebras," Adv. in Math. 180 (2003) no. 2, 596-647, arXiv:math/0203010.

[70] J. C. Baez, "The Octonions," Bull. Am. Math. Soc. 39 (2002) 145-205, arXiv:math/0105155 [math-ra].

[71] A. Hurwitz, "Uber die komposition der quadratishen formen von beliebig vielen variabeln," Nachr. Ges. Wiss. Gottingen (1898) 309-316.

[72] R. Moufang, "Alternativkörper und der satz vom vollsta ndigen vierseit," Abh. Math. Sem. Hamburg 9 (1933) 207-222. 
[73] P. Jordan, "Über eine nicht-desarguessche ebene projektive geometrie," Abh. Math. Sem. Hamburg 16 (1949) 74-76.

[74] C. Chevalley and R. D. Schafer, "The exceptional simple lie algebras $f_{4}$ and $e_{6}$," Proc. Nat. Acad. Sci. USA (1950) 137-141.

[75] A. Anastasiou, L. Borsten, M. J. Duff, L. J. Hughes, and S. Nagy, "A magic pyramid of supergravities," JHEP 1404 (2014) 178, arXiv:1312.6523 [hep-th].

[76] M. Gunaydin, "Exceptional Realizations of Lorentz Group: Supersymmetries and Leptons," Nuovo Cim. A29 (1975) 467.

[77] M. Gunaydin, "Octonionic Hilbert Spaces, the Poincare Group and SU(3)," J.Math.Phys. 17 (1976) 1875.

[78] F. Gursey, "Octonionic Structures in Particle Physics," Lect.Notes Phys. 94 (1979) 508-521.

[79] M. Gunaydin, "Quadratic Jordan Formulation Of Quantum Mechanics And Construction Of Lie (Super)Algebras From Jordan (Super)Algebras," in International colloquium on group theoretical methods in physics, vol. 10, p. 18. Israel Grp.Th.Meth., 1979.

[80] T. Kugo and P. K. Townsend, "Supersymmetry and the division algebras," Nucl. Phys. B221 (1983) 357.

[81] A. Sudbery, "Division algebras, (pseudo)orthogonal groups, and spinors," J. Phys. A17 (1984) no. 5, 939-955.

[82] F. Gursey, "Superpoincare Groups and Division Algebras," Mod.Phys.Lett. A2 (1987) 967.

[83] M. B. Green, J. H. Schwarz, and E. Witten, Superstring Theory vol. 1: Introduction. Cambridge Monographs on Mathematical Physics. Cambridge University Press, Cambridge, UK, 1987. 469 p.

[84] J. M. Evans, "Supersymmetric Yang-Mills theories and division algebras," Nucl. Phys. B298 (1988) 92

[85] M. J. Duff, "Supermembranes: The First Fifteen Weeks," Class.Quant.Grav. 5 (1988) 189.

[86] M. Blencowe and M. J. Duff, "Supermembranes and the Signature of Space-time," Nucl.Phys. B310 (1988) 387.

[87] M. Gunaydin, "Generalized conformal and superconformal group actions and Jordan algebras," Mod.Phys.Lett. A8 (1993) 1407-1416, arXiv:hep-th/9301050 [hep-th].

[88] N. Berkovits, "A Ten-dimensional superYang-Mills action with off-shell supersymmetry," Phys.Lett. B318 (1993) 104-106, arXiv:hep-th/9308128 [hep-th].

[89] C. A. Manogue and J. Schray, "Finite Lorentz transformations, automorphisms, and division algebras," J. Math. Phys. 34 (1993) 3746-3767, arXiv:hep-th/9302044.

[90] J. M. Evans, "Auxiliary fields for superYang-Mills from division algebras," Lect.Notes Phys. 447 (1995) 218-223, arXiv:hep-th/9410239 [hep-th].

[91] J. Schray and C. A. Manogue, "Octonionic representations of Clifford algebras and triality," Found. Phys. 26 (1996) no. 1, 17-70, arXiv: hep-th/9407179. 
[92] F. Gürsey and C.-H. Tze, On the role of division, Jordan and related algebras in particle physics. World Scientific, London, 1996. http://www.worldscientific.com/doi/abs/10.1142/9789812819857.

[93] C. A. Manogue and T. Dray, "Dimensional reduction," Mod.Phys.Lett. A14 (1999) 99-104, arXiv:hep-th/9807044 [hep-th].

[94] M. Günaydin, K. Koepsell, and H. Nicolai, "Conformal and quasiconformal realizations of exceptional Lie groups," Commun. Math. Phys. 221 (2001) 57-76, arXiv:hep-th/0008063.

[95] F. Toppan, "On the octonionic M-superalgebra," in Sao Paulo 2002, Integrable theories, solitons and duality. 2002. arXiv:hep-th/0301163.

[96] M. Günaydin and O. Pavlyk, "Generalized spacetimes defined by cubic forms and the minimal unitary realizations of their quasiconformal groups," JHEP 08 (2005) 101, arXiv:hep-th/0506010.

[97] L. Borsten, D. Dahanayake, M. J. Duff, H. Ebrahim, and W. Rubens, "Black Holes, Qubits and Octonions," Phys. Rep. 471 (2009) no. 3-4, 113-219, arXiv:0809.4685 [hep-th].

[98] J. C. Baez and J. Huerta, "Division algebras and supersymmetry i," in Superstrings, Geometry, Topology, and $C^{*}$-Algebras, eds. R. Doran, G. Friedman and J. Rosenberg, Proc. Symp. Pure Math, vol. 81, pp. 65-80. 2009. arXiv:0909.0551 [hep-th].

[99] J. C. Baez and J. Huerta, "Division Algebras and Supersymmetry II," Adv. Theor. Math. Phys. 15 (2011) no. 5, 1373-1410, arXiv:1003.3436 [hep-th].

[100] J. Huerta, "Division Algebras, Supersymmetry and Higher Gauge Theory," arXiv:1106.3385 [math-ph].

[101] J. Huerta, "Division Algebras and Supersymmetry III," Adv.Theor.Math.Phys. 16 (2012) 1485-1589, arXiv:1109.3574 [hep-th].

[102] A. Anastasiou, L. Borsten, M. J. Duff, L. J. Hughes, and S. Nagy, "An octonionic formulation of the M-theory algebra," JHEP 1411 (2014) 022, arXiv:1402.4649 [hep-th].

[103] J. Huerta, "Division Algebras and Supersymmetry IV," arXiv:1409.4361 [hep-th].

[104] A. Marrani and P. Truini, "Exceptional Lie Algebras, SU(3) and Jordan Pairs Part 2: Zorn-type Representations," J.Phys. A47 (2014) 265202, arXiv:1403.5120 [math-ph].

[105] K. Chung and A. Sudbery, "Octonions and the Lorentz and Conformal Groups of Ten-dimensional Space-time," Phys.Lett. B198 (1987) 161.

[106] D. B. Fairlie and C. A. Manogue, "A Parametrization of the Covariant Superstring," Phys.Rev. D36 (1987) 475.

[107] C. A. Manogue and A. Sudbery, "General Solutions of Covariant Superstring Equations of Motion," Phys.Rev. D40 (1989) 4073.

[108] J. Schray, "The General classical solution of the superparticle," Class.Quant.Grav. 13 (1996) 27-38, arXiv:hep-th/9407045 [hep-th].

[109] T. Dray, J. Janesky, and C. A. Manogue, "Octonionic hermitian matrices with non-real eigenvalues," Advances in Applied Clifford Algebras 10 (2000) no. 2, 193-216. 
[110] A. Anastasiou, L. Borsten, M. J. Duff, L. J. Hughes, and S. Nagy, "Super Yang-Mills, division algebras and triality," JHEP 1408 (2014) 080, arXiv: 1309.0546 [hep-th].

[111] C. Hull, "Strongly coupled gravity and duality," Nucl.Phys. B583 (2000) 237-259, arXiv:hep-th/0004195 [hep-th].

[112] C. Hull, "Symmetries and compactifications of (4,0) conformal gravity," JHEP 0012 (2000) 007, arXiv:hep-th/0011215 [hep-th].

[113] C. Hull, "Conformal nongemetric gravity in six-dimensions and M theory above the Planck energy," Class.Quant.Grav. 18 (2001) 3233-3240, arXiv:hep-th/0011171 [hep-th].

[114] Y.-t. Huang and A. E. Lipstein, "Amplitudes of 3D and 6D Maximal Superconformal Theories in Supertwistor Space," JHEP 1010 (2010) 007, arXiv:1004.4735 [hep-th].

[115] B. Czech, Y.-t. Huang, and M. Rozali, "Chiral three-point interactions in 5 and 6 dimensions," JHEP 1210 (2012) 143, arXiv:1110.2791 [hep-th].

[116] T. Bargheer, S. He, and T. McLoughlin, "New Relations for Three-Dimensional Supersymmetric Scattering Amplitudes," Phys.Rev.Lett. 108 (2012) 231601, arXiv:1203.0562 [hep-th].

[117] Y.-t. Huang and H. Johansson, "Equivalent $\mathrm{D}=3$ Supergravity Amplitudes from Double Copies of Three-Algebra and Two-Algebra Gauge Theories," Phys.Rev.Lett. 110 (2013) 171601. arXiv:1210.2255 [hep-th].

[118] J. Bagger and N. Lambert, "Modeling Multiple M2's," Phys.Rev. D75 (2007) 045020, arXiv:hep-th/0611108 [hep-th].

[119] A. Gustavsson, "Algebraic structures on parallel M2-branes," Nucl.Phys. B811 (2009) 66-76, arXiv:0709.1260 [hep-th].

[120] J. Bagger and N. Lambert, "Gauge symmetry and supersymmetry of multiple M2-branes," Phys.Rev. D77 (2008) 065008, arXiv:0711.0955 [hep-th].

[121] L. Borsten, "On the $d=6, \mathcal{N}=(4,0)$ theory." to appear, 2016. 